\begin{tabular}{|c|l|}
\hline Title & Equation of motion for point vortices in multiply connected circular domains \\
\hline Author(s) & Sakajo, Takashi \\
\hline Citation & $\begin{array}{l}\text { Proceedings of the Royal Society A : Mathematical, Physical \& Engineering Sciences, 465(2108), 2589-2611 } \\
\text { https://doi.org/40.1098/spa.2009.0070 }\end{array}$ \\
\hline Issue Date & 2009-08-08 \\
\hline Doc URL & http://hdl.handle.net/2115/43389 \\
\hline Type & article (author version) \\
\hline File Information & PRSA 465-2108_2589-2611.pdf \\
\hline
\end{tabular}

Instructions for use 


\title{
Equation of motion for point vortices in multiply connected circular domains
}

\author{
Takashi SAKAJO \\ Department of mathematics, Hokkaido University, \\ PRESTO, Japan Science and Technology Agency
}

\begin{abstract}
The paper gives the equation of motion for $N$ point vortices in a bounded planar multiply connected domain inside the unit circle that contains many circular obstacles, called the circular domain. The velocity field induced by the point vortices is described in terms of the Schottky-Klein prime function associated with the circular domain. The explicit representation of the equation enables us not only to solve the Euler equations through the point-vortex approximation numerically, but also to investigate the interactions between localized vortex structures in the circular domain. As an application of the equation, we consider the motion of two point vortices with the unit strength of the opposite signs. When the multiply connected domain is symmetric with respect to the real axis, the motion of the two point vortices is reduced to that of a single point vortex in a multiply connected semi-circle, which we investigate in detail.
\end{abstract}

\section{Introduction}

The study of incompressible and inviscid flows in planar multiply connected domains is not only one of the fundamental subjects in the field of mathematical fluid dynamics, but it also contributes toward understanding of geophysical flows with many islands and artificial obstacles such as lakes, inland seas and coastal regions. This is because these regions are mathematically represented by multiply connected domains.

The motion of the incompressible and inviscid flow is described by the two-dimensional Euler equations. According to Kelvin's theorem, the circulation is conserved along the path of a fluid particle and thus the vorticity neither generates nor disappears during its evolution. Hence in order to solve the Euler equations, we have only to investigate the evolution of the non-zero vorticity domain at the initial moment. Based on this observation, we discretize the initial nonzero vorticity domain with a set of $N$ points, called point vortices, whose strengths are determined by the circulations around these points. Then we track the evolutions of the $N$ point vortices. This discretization method for the Euler equations is known as the vortex method[2], which reduces the Euler equations to a system of ordinary differential equations for the $N$ point vortices. The pointvortex system is often used as a simple mathematical model to describe the interactions between localized vortex structures where the vorticity concentrates in small regions. The readers can find many results and their references of this topic in the books of Newton[11] and Saffman[12].

The point-vortex system is formulated as a Hamiltonian dynamical system, whose Hamiltonian is conventionally called the Kirchhoff-Routh function. Suppose that the $N$ point vortices $\left\{z_{\lambda} \mid \lambda=\right.$ $1, \ldots, N\}$ with the strengths $\left\{\Gamma_{\lambda} \mid \lambda=1, \ldots, N\right\}$ are in a domain $\mathcal{D} \subset \mathbb{C}$. Then the Kirchhoff-Routh 
function is represented by

$$
H\left(z_{1}, z_{1}^{*}, \ldots, z_{N}, z_{N}^{*}\right)=\frac{1}{2} \sum_{\lambda=1}^{N} \sum_{\alpha \neq \lambda}^{N} \Gamma_{\lambda} \Gamma_{\alpha} G\left(z_{\lambda} ; z_{\alpha}\right)-\frac{1}{2} \sum_{\lambda=1}^{N} \Gamma_{\lambda}^{2} \mathcal{R}\left(z_{\lambda} ; z_{\lambda}^{*}\right) \equiv H_{G}+H_{R},
$$

where $z_{\lambda}^{*}$ denotes the complex conjugation of $z_{\lambda}$. The function $G(z ; w)$ is the hydrodynamic Green's function satisfying the following Poisson equation,

$$
\Delta G(z ; w)+\delta(|z-w|)=0 \quad \text { in } \quad \mathcal{D},
$$

with a certain boundary condition imposed on $\partial \mathcal{D}$, in which the $\delta$-function represents a source at $w \in \mathcal{D}$. The function $\mathcal{R}(z ; w)$ is derived from the Green's function by

$$
\mathcal{R}(z ; w)=-G(z ; w)-\frac{1}{2 \pi} \log |z-w|,
$$

which is called the Robin function [7]. Note that $H_{G}$ and $H_{R}$ represent the vortex-vortex interaction and the vortex-boundary interaction respectively. $\operatorname{Lin}[8,9]$ showed how to derive the equation for the $N$ point vortices from the Kirchhoff-Routh function, which is stated as follows[11]:

Theorem 1 Let $H\left(z_{1}, z_{1}^{*}, z_{2}, z_{2}^{*}, \cdots, z_{N}, z_{N}^{*}\right)$ be the Kirchhoff-Routh function for the $N$ point vortices $\left\{z_{\lambda} \mid \lambda=1, \ldots, N\right\}$ with the strengths $\left\{\Gamma_{\lambda} \mid \lambda=1, \ldots, N\right\}$ in a domain $\mathcal{D} \subset \mathbb{C}$. Then the equation of motion for the $N$ point vortices is given by

$$
\Gamma_{\lambda} \frac{d z_{\lambda}}{d t}=-2 i \frac{\partial H}{\partial z_{\lambda}^{*}},
$$

where $i=\sqrt{-1}$ denotes the complex unit.

The Kirchhoff-Routh functions for the unbounded plane, the surface of a sphere and some simple domains with boundaries have been obtained[11, 12]. On the other hand, Crowdy and Marshall[3] recently considered a bounded multiply connected domain inside the unit circle with many circular boundaries, which is called the circular domain, for which they gave an analytic representation of the Kirchhoff-Routh function. It has been applied to describe the steady irrotational uniform flow past many cylindrical obstacles in a plane[5] and to compute the lift on the cylindrical obstacles[6].

Crowdy and Marshall[4] have investigated the motion of a single point vortex, which is equivalent to a contour line of the Kirchhoff-Routh function, since the Hamiltonian dynamical system (3) for $N=1$ is always integrable. On the other hand, the motion of more than one point vortex is no longer integrable due to lack of special symmetry of the circular domain, and it has not been investigated well. This is due to the lack of an explicit representation of the equation for the $N$ point vortices. Thus the primary purpose of the present paper is deriving the equation for the $N$-point vortices in the circular domain from the Kirchhoff-Routh function given by Crowdy and Marshall[3] and Lin's theorem. Then we make use of the equation to investigate the motion of two point vortices with the unit strength of the opposite signs in the circular domain.

The paper consists of four sections. In the next section, we derive the equation of the $N$ point vortices in the multiply connected circular domain from the Kirchhoff-Routh function given by Crowdy and Marshall[3]. In $\S 3$, we investigate the motion of two point vortices in a circular domain that is symmetric with respect to the real axis. Owing to the symmetry, it is reduced to the motion of a single point vortex in the multiply connected upper semi-circle. The last section gives a summary and concluding remarks. 


\section{Equation for the $N$ point vortices in circular domains}

We introduce a special multiply connected domain $D_{\zeta}$ in the complex $\zeta$-plane, called the circular domain, inside the unit circle $|\zeta| \leq 1$ with $M$ circular obstacles. The circular domain is regarded as a canonical multiply connected domain since it is mathematically shown that for any bounded domain with $M$ holes, there exists a conformal mapping that maps the domain to a circular domain with $M$ circular obstacles[10]. Let $C_{0}$ denote the unit circle and $\left\{C_{i} \mid i=1, \ldots, M\right\}$ represent the boundaries of the disjoint $M$ circular obstacles inside the unit circle, whose centers and radii are denoted by $\delta_{i} \in D_{\zeta}$ and $q_{i} \in \mathbb{R}$. The conjugation map $\phi_{i}(\zeta)$ with respect to the unit circle associated with the circle $C_{i}$ is given by

$$
\phi_{i}(\zeta)=\delta_{i}^{*}+\frac{q_{i}^{2}}{\zeta-\delta_{i}}, \quad i=1, \ldots, M
$$

with which we define the Möbius maps $\vartheta_{i}(\zeta)$ as

$$
\vartheta_{i}(\zeta) \equiv \phi_{i}^{*}\left(\zeta^{-1}\right)=\delta_{i}+\frac{q_{i}^{2} \zeta}{1-\delta_{i}^{*} \zeta} .
$$

Here we use the notations $(\phi(\zeta))^{*}=\phi^{*}\left(\zeta^{*}\right)$ and $\left(\phi\left(\zeta^{*}\right)\right)^{*}=\phi^{*}(\zeta)$ for the conjugation of the map.

The infinite free group of maps generated by the basic Möbius maps $\vartheta_{i}(\zeta)$ and their inverses $\vartheta_{i}^{-1}(\zeta)$ for $i=1, \ldots, M$ is called the Schottky group, which is denoted by $\Theta$. Note that every element $\theta_{i}(\zeta)$ in the Schottky group is represented by a composition of these $2 M$ basic maps. Let $\Theta^{\prime \prime}$ represent a subset of the Schottky group $\Theta$ that excludes the identity map and all inverse mappings. Then the Schottky-Klein prime function $\omega(\zeta, \alpha)$ is defined as follows[1]:

$$
\omega(\zeta, \alpha)=(\zeta-\alpha) \omega^{\prime}(\zeta, \alpha), \quad \omega^{\prime}(\zeta, \alpha)=\prod_{\theta_{i} \in \Theta^{\prime \prime}} \frac{\left(\theta_{i}(\zeta)-\alpha\right)\left(\theta_{i}(\alpha)-\zeta\right)}{\left(\theta_{i}(\zeta)-\zeta\right)\left(\theta_{i}(\alpha)-\alpha\right)}, \quad \zeta, \alpha \in D_{\zeta} .
$$

For the unit circle $|\zeta| \leq 1$, the only element in the Schottky group is the identity map, since there is no obstacle inside. Then the subset $\Theta^{\prime \prime}$ is the empty set from the definition. For the doubly connected concentric annulus $\{q<|\zeta|<1\}$, there is only one Möbius map $\vartheta_{1}(\zeta)=q^{2} \zeta$ associated with the inner boundary $|\zeta|=q$. Since the Schottky group consists of all compositions of $\vartheta_{1}(\zeta)=q^{2} \zeta$ and its inverse $\vartheta_{1}^{-1}(\zeta)=q^{-2} \zeta$, any element in the subset $\Theta^{\prime \prime}$ is represented by $\theta_{k}(\zeta)=q^{2 k} \zeta$ for $k>1$.

Crowdy and Marshall[3] gave the Green's function $G(\zeta ; \alpha)$ and the Robin function $\mathcal{R}\left(\alpha ; \alpha^{*}\right)$ for the circular domain $D_{\zeta}$ as follows:

$$
G(\zeta ; \alpha)=-\frac{1}{2 \pi} \log \left|\frac{1}{\alpha} \frac{\omega(\zeta, \alpha)}{\omega\left(\zeta, \alpha^{*-1}\right)}\right|, \quad \mathcal{R}\left(\alpha ; \alpha^{*}\right)=\frac{1}{4 \pi} \log \left|\frac{\omega^{\prime}(\alpha, \alpha) \omega^{\prime *}\left(\alpha^{-1}, \alpha^{-1}\right)}{\alpha^{2} \omega\left(\alpha, \alpha^{*-1}\right) \omega^{*}\left(\alpha^{-1}, \alpha^{*}\right)}\right| .
$$

The Kirchhoff-Routh function (1) with the Green's function and the Robin function enables us to derive the equation for the $N$ point vortices in the circular domain $D_{\zeta}$ with Theorem 1 . The following theorem is one of the main results of this paper.

Theorem 2 Let $\left\{z_{\lambda} \mid \lambda=1, \ldots, N\right\}$ denote the positions of $N$ point vortices with the strengths $\left\{\Gamma_{\lambda} \mid \lambda=1, \ldots, N\right\}$ in the circular domain $D_{\zeta} \subset \mathbb{C}$. Then the motion of the point vortices is described by

$$
\frac{d z_{\lambda}}{d t}=\frac{i}{2 \pi} \sum_{\alpha \neq \lambda}^{N} \Gamma_{\alpha}\left(\frac{\omega_{\zeta}^{*}\left(z_{\lambda}^{*}, z_{\alpha}^{*}\right)}{\omega^{*}\left(z_{\lambda}^{*}, z_{\alpha}^{*}\right)}-\frac{\omega_{\zeta}^{*}\left(z_{\lambda}^{*}, z_{\alpha}^{-1}\right)}{\omega^{*}\left(z_{\lambda}^{*}, z_{\alpha}^{-1}\right)}\right)-\frac{i}{2 \pi} \Gamma_{\lambda} \frac{\omega_{\zeta}^{*}\left(z_{\lambda}^{*}, z_{\lambda}^{-1}\right)}{\omega^{*}\left(z_{\lambda}^{*}, z_{\lambda}^{-1}\right)}, \quad \lambda=1, \ldots, N,
$$

in which $\omega_{\zeta}(\zeta, \alpha)=\frac{\partial}{\partial \zeta} \omega(\zeta, \alpha)$ and $\omega_{\zeta}^{*}\left(\zeta^{*}, \alpha^{*}\right)=\left(\omega_{\zeta}(\zeta, \alpha)\right)^{*}$. 
The two terms in the summation represent the contributions from the point vortex at $z_{\alpha}$ and its conjugation point with respect to the unit circle at $z_{\alpha}^{*-1}$, while the last term is the velocity field induced by the point vortex at the conjugation point $z_{\lambda}^{*-1}$ of $z_{\lambda}$. Since the function $\omega_{\zeta}(\zeta, \alpha) / \omega(\zeta, \alpha)$ plays a key role in the equation (5), the following expression is useful.

Proposition 1 The function $\omega_{\zeta}(\zeta, \alpha) / \omega(\zeta, \alpha)$ is given by

$$
\frac{\omega_{\zeta}(\zeta, \alpha)}{\omega(\zeta, \alpha)}=\frac{1}{\zeta-\alpha}+\sum_{\theta_{i} \in \Theta^{\prime \prime}}\left\{\frac{\theta_{i}^{\prime}(\zeta)(\alpha-\zeta)}{\left(\theta_{i}(\zeta)-\alpha\right)\left(\theta_{i}(\zeta)-\zeta\right)}+\frac{\theta_{i}(\alpha)-\theta_{i}(\zeta)}{\left(\theta_{i}(\alpha)-\zeta\right)\left(\theta_{i}(\zeta)-\zeta\right)}\right\}
$$

in which $\theta_{i}^{\prime}(\zeta)=\frac{d}{d \zeta} \theta_{i}(\zeta)$

Proof: The calculation is straightforward. Let us write $\omega^{\prime}(\zeta, \alpha)=\prod_{\theta_{i} \in \Theta^{\prime \prime}} \Omega^{(i)}(\zeta, \alpha)$, where

$$
\Omega^{(i)}(\zeta, \alpha)=\frac{\left(\theta_{i}(\zeta)-\alpha\right)\left(\theta_{i}(\alpha)-\zeta\right)}{\left(\theta_{i}(\zeta)-\zeta\right)\left(\theta_{i}(\alpha)-\alpha\right)}
$$

Since we have

$$
\frac{\omega_{\zeta}(\zeta, \alpha)}{\omega(\zeta, \alpha)}=\frac{d}{d \zeta} \log \omega(\zeta, \alpha)=\frac{d}{d \zeta}\left(\log (\zeta-\alpha)+\log \omega^{\prime}(\zeta, \alpha)\right)=\frac{1}{\zeta-\alpha}+\frac{d}{d \zeta} \sum_{\theta_{i} \in \Theta^{\prime \prime}} \log \Omega^{(i)}(\zeta, \alpha),
$$

the proof is finished by

$$
\begin{aligned}
\frac{d}{d \zeta} \log \Omega^{(i)}(\zeta, \alpha) & =\frac{\theta_{i}^{\prime}(\zeta)}{\theta_{i}(\zeta)-\alpha}-\frac{1}{\theta_{i}(\alpha)-\zeta}-\frac{\theta_{i}^{\prime}(\zeta)-1}{\theta_{i}(\zeta)-\zeta} \\
& =\frac{\theta_{i}^{\prime}(\zeta)(\alpha-\zeta)}{\left(\theta_{i}(\zeta)-\alpha\right)\left(\theta_{i}(\zeta)-\zeta\right)}+\frac{\theta_{i}(\alpha)-\theta_{i}(\zeta)}{\left(\theta_{i}(\alpha)-\zeta\right)\left(\theta_{i}(\zeta)-\zeta\right)}
\end{aligned}
$$

Owing to this proposition, we write down the explicit representations of $\omega_{\zeta}(\zeta, \alpha) / \omega(\zeta, \alpha)$ for the simply connected unit disk $|\zeta| \leq 1$, and the doubly connected concentric annulus $\{\zeta|q<| \zeta \mid<1\}$. For the unit circle, we have $\omega(\zeta, \alpha)=(\zeta-\alpha)$, since the subset $\Theta^{\prime \prime}$ is the empty set, which leads to

$$
\frac{\omega_{\zeta}(\zeta, \alpha)}{\omega(\zeta, \alpha)}=\frac{1}{\zeta-\alpha} .
$$

Regarding the doubly connected concentric annulus, any element in $\Theta^{\prime \prime}$ is represented by $\theta_{k}(\zeta)=$ $q^{2 k} \zeta$ for $k>1$. Hence, it follows from Proposition 1 and

$$
\begin{aligned}
\frac{\theta_{k}^{\prime}(\zeta)(\alpha-\zeta)}{\left(\theta_{k}(\zeta)-\alpha\right)\left(\theta_{k}(\zeta)-\zeta\right)}+\frac{\theta_{i}(\alpha)-\theta_{i}(\zeta)}{\left(\theta_{i}(\alpha)-\zeta\right)\left(\theta_{i}(\zeta)-\zeta\right)} & =\frac{q^{2 k}(\alpha-\zeta)}{\left(q^{2 k} \zeta-\alpha\right)\left(q^{2 k} \zeta-\zeta\right)}+\frac{q^{2 k}(\alpha-\zeta)}{\left(q^{2 k} \alpha-\zeta\right)\left(q^{2 k} \zeta-\zeta\right)} \\
& =\frac{q^{2 k}\left(\alpha^{2}-\zeta^{2}\right)}{\left(q^{2 k} \zeta-\alpha\right)\left(q^{2 k} \alpha-\zeta\right)} \frac{1}{\zeta} .
\end{aligned}
$$

that we have

$$
\frac{\omega_{\zeta}(\zeta, \alpha)}{\omega(\zeta, \alpha)}=\frac{1}{\zeta-\alpha}+\sum_{k=1}^{\infty} \frac{q^{2 k}\left(\alpha^{2}-\zeta^{2}\right)}{\left(q^{2 k} \zeta-\alpha\right)\left(q^{2 k} \alpha-\zeta\right)} \frac{1}{\zeta} .
$$

In what follows, we give the proof of Theorem 2. First, let us show some basic properties for the Schottky-Klein prime function $\omega(\zeta, \alpha)$. 
Lemma 1 The Schottky-Klein-Prime function $\omega(\zeta, \alpha)$ satisfies the following.
(i). $\omega^{*}\left(\zeta^{-1}, \alpha^{-1}\right)=-\frac{1}{\zeta \alpha} \omega(\zeta, \alpha)$
(ii). $\omega(\zeta, \alpha)=-\omega(\alpha, \zeta)$,
(iii). $\omega^{\prime}(\zeta, \alpha)=\omega^{\prime}(\alpha, \zeta)$,
(iv). For $z \in D_{\zeta}, \omega_{\zeta}^{\prime}(z, z)=\omega_{\alpha}^{\prime}(z, z)=0$,
(v). For $z \in D_{\zeta}, \omega_{\zeta}\left(z^{*-1}, z\right)=-\omega_{\alpha}\left(z, z^{*-1}\right)$.

Proof: Since the properties (i)-(iii) have already been confirmed in [3], we prove the last two properties. Differentiating $\log \omega^{\prime}$ with respect to $\zeta$ and $\alpha$ as in the proof of Proposition 1, we have

$$
\begin{aligned}
& \frac{\omega_{\zeta}^{\prime}(\zeta, \alpha)}{\omega^{\prime}(\zeta, \alpha)}=\sum_{\theta_{i} \in \Theta^{\prime \prime}} \frac{\theta_{i}^{\prime}(\zeta)}{\theta_{i}(\zeta)-\alpha}-\frac{1}{\theta_{i}(\alpha)-\zeta}-\frac{\theta_{i}^{\prime}(\zeta)-1}{\theta_{i}(\zeta)-\zeta} \\
& \frac{\omega_{\alpha}^{\prime}(\zeta, \alpha)}{\omega^{\prime}(\zeta, \alpha)}=\sum_{\theta_{i} \in \Theta^{\prime \prime}} \frac{-1}{\theta_{i}(\zeta)-\alpha}+\frac{\theta_{i}^{\prime}(\alpha)}{\theta_{i}(\alpha)-\zeta}-\frac{\theta_{i}^{\prime}(\alpha)-1}{\theta_{i}(\alpha)-\alpha}
\end{aligned}
$$

Substituting $\zeta=\alpha=z \in D_{\zeta}$, we have $\omega_{\zeta}^{\prime}(z, z)=\omega_{\alpha}^{\prime}(z, z)=0$ due to $\omega^{\prime}(z, z)=1$.

Regarding (v), we first show $\omega_{\zeta}^{\prime}\left(z^{*-1}, z\right)=\omega_{\alpha}^{\prime}\left(z, z^{*-1}\right)$ for $z \in D_{\zeta}$. It is easy to see from (10) and (11) that

$$
\frac{\omega_{\zeta}^{\prime}\left(z^{*-1}, z\right)}{\omega^{\prime}\left(z^{*-1}, z\right)}=\sum_{\theta_{i} \in \Theta^{\prime \prime}} \frac{\theta_{i}^{\prime}\left(z^{*-1}\right)}{\theta_{i}\left(z^{*-1}\right)-z}-\frac{1}{\theta_{i}(z)-z^{*-1}}-\frac{\theta_{i}^{\prime}\left(z^{*-1}\right)-1}{\theta_{i}\left(z^{*-1}\right)-z^{*-1}}=\frac{\omega_{\alpha}\left(z, z^{*-1}\right)}{\omega^{\prime}\left(z, z^{*-1}\right)} .
$$

Since $\omega^{\prime}\left(z^{*-1}, z\right)=\omega^{\prime}\left(z, z^{*-1}\right)$ due to (iii), we have $\omega_{\zeta}^{\prime}\left(z^{*-1}, z\right)=\omega_{\alpha}^{\prime}\left(z, z^{*-1}\right)$. Then it follows from

$$
\omega_{\zeta}(\zeta, \alpha)=\omega^{\prime}(\zeta, \alpha)+(\zeta-\alpha) \omega_{\zeta}^{\prime}(\zeta, \alpha), \quad \omega_{\alpha}(\zeta, \alpha)=-\omega^{\prime}(\zeta, \alpha)+(\zeta-\alpha) \omega_{\alpha}^{\prime}(\zeta, \alpha)
$$

that we have

$$
\begin{aligned}
\omega_{\alpha}\left(z, z^{*-1}\right) & =-\omega^{\prime}\left(z, z^{*-1}\right)+\left(z-z^{*-1}\right) \omega_{\alpha}^{\prime}\left(z, z^{*-1}\right) \\
& =-\omega^{\prime}\left(z^{*-1}, z\right)-\left(z^{*-1}-z\right) \omega_{\zeta}^{\prime}\left(z^{*-1}, z\right)=-\omega_{\zeta}\left(z^{*-1}, z\right) .
\end{aligned}
$$

Now, we calculate the right-hand side of (3). First, we deal with the contribution from the vortex-vortex interaction part $H_{G}$. Let us rewrite the Green's function $G\left(z_{\lambda} ; z_{\alpha}\right)$ as

$$
G\left(z_{\lambda} ; z_{\alpha}\right)=-\frac{1}{4 \pi} \log \left(\frac{\omega\left(z_{\lambda}, z_{\alpha}\right)}{z_{\alpha} \omega\left(z_{\lambda}, z_{\alpha}^{*-1}\right)}\right)\left(\frac{\omega^{*}\left(z_{\lambda}^{*}, z_{\alpha}^{*}\right)}{z_{\alpha}^{*} \omega^{*}\left(z_{\lambda}^{*}, z_{\alpha}^{-1}\right)}\right) .
$$

The terms in $H_{G}$ that contain the variable $z_{\lambda}$ are given by

$$
\frac{1}{2} \sum_{\alpha \neq \lambda}\left\{\Gamma_{\lambda} \Gamma_{\alpha} G\left(z_{\lambda} ; z_{\alpha}\right)+\Gamma_{\alpha} \Gamma_{\lambda} G\left(z_{\alpha} ; z_{\lambda}\right)\right\}=\sum_{\alpha \neq \lambda} \Gamma_{\lambda} \Gamma_{\alpha} G\left(z_{\lambda} ; z_{\alpha}\right) .
$$


Since the derivative of $G\left(z_{\lambda} ; z_{\alpha}\right)$ with respect to $z_{\lambda}^{*}$ becomes

$$
\frac{\partial G}{\partial z_{\lambda}^{*}}=-\frac{1}{4 \pi} \frac{\omega_{\zeta}^{*}\left(z_{\lambda}^{*}, z_{\alpha}^{*}\right)}{\omega^{*}\left(z_{\lambda}^{*}, z_{\alpha}^{*}\right)}+\frac{1}{4 \pi} \frac{\omega_{\zeta}^{*}\left(z_{\lambda}^{*}, z_{\alpha}^{-1}\right)}{\omega^{*}\left(z_{\lambda}^{*}, z_{\alpha}^{-1}\right)},
$$

we have

$$
-2 \mathrm{i} \frac{\partial H_{G}}{\partial z_{\lambda}^{*}}=\frac{\mathrm{i}}{2 \pi} \sum_{\alpha \neq \lambda}^{N} \Gamma_{\alpha} \Gamma_{\lambda}\left(\frac{\omega_{\zeta}^{*}\left(z_{\lambda}^{*}, z_{\alpha}^{*}\right)}{\omega^{*}\left(z_{\lambda}^{*}, z_{\alpha}^{*}\right)}-\frac{\omega_{\zeta}^{*}\left(z_{\lambda}^{*}, z_{\alpha}^{-1}\right)}{\omega^{*}\left(z_{\lambda}^{*}, z_{\alpha}^{-1}\right)}\right) .
$$

Next, we consider the contribution from the vortex-boundary interaction part $H_{R}$. The following lemma gives us a simpler expression of the Robin function.

Lemma 2 The Robin function $\mathcal{R}\left(\alpha ; \alpha^{*}\right)$ is expressed as

$$
\mathcal{R}\left(\alpha ; \alpha^{*}\right)=\frac{1}{2 \pi} \log \left|\frac{\omega^{\prime}(\alpha, \alpha)}{|\alpha| \omega\left(\alpha, \alpha^{*-1}\right)}\right| .
$$

Proof It follows from (i) of Lemma 1 that we have

$$
-\frac{1}{\zeta \alpha}(\zeta-\alpha) \omega^{\prime}(\zeta, \alpha)=\omega^{*}\left(\zeta^{-1}, \alpha^{-1}\right)=\frac{\alpha-\zeta}{\zeta \alpha} \omega^{\prime *}\left(\zeta^{-1}, \alpha^{-1}\right) .
$$

Thus we have $\omega^{\prime *}\left(\alpha^{-1}, \alpha^{-1}\right)=\omega^{\prime}(\alpha, \alpha)$. Then the Robin function $\mathcal{R}$ is rewritten as

$$
\begin{aligned}
\mathcal{R}\left(\alpha ; \alpha^{*}\right) & =\frac{1}{4 \pi} \log \left|\frac{\omega^{\prime}(\alpha, \alpha) \omega^{\prime *}\left(\alpha^{-1}, \alpha^{-1}\right)}{\alpha^{2} \omega\left(\alpha, \alpha^{*-1}\right) \omega^{*}\left(\alpha^{-1}, \alpha^{*}\right)}\right| \\
& =\frac{1}{4 \pi} \log \left|\frac{\omega^{\prime}(\alpha, \alpha) \omega^{\prime}(\alpha, \alpha)}{\alpha^{2} \omega\left(\alpha, \alpha^{*-1}\right) \cdot-\frac{\alpha^{*}}{\alpha} \omega\left(\alpha, \alpha^{*-1}\right)}\right|=\frac{1}{2 \pi} \log \left|\frac{\omega^{\prime}(\alpha, \alpha)}{|\alpha| \omega\left(\alpha, \alpha^{*-1}\right)}\right| .
\end{aligned}
$$

Due to this lemma, $\mathcal{R}\left(z_{\lambda} ; z_{\lambda}^{*}\right)$ becomes

$$
\mathcal{R}\left(z_{\lambda} ; z_{\lambda}^{*}\right)=\frac{1}{2 \pi} \log \left|\frac{\omega^{\prime}\left(z_{\lambda}, z_{\lambda}\right)}{\left|z_{\lambda}\right| \omega\left(z_{\lambda}, z_{\lambda}^{*-1}\right)}\right|=\frac{1}{4 \pi} \log \frac{1}{\left|z_{\lambda}\right|^{2}}\left(\frac{\omega^{\prime}\left(z_{\lambda}, z_{\lambda}\right)}{\omega\left(z_{\lambda}, z_{\lambda}^{*-1}\right)}\right)\left(\frac{\omega^{\prime *}\left(z_{\lambda}^{*}, z_{\lambda}^{*}\right)}{\omega^{*}\left(z_{\lambda}^{*}, z_{\lambda}^{-1}\right)}\right) .
$$

Differentiating it with respect to $z_{\lambda}^{*}$, we have

$$
\begin{aligned}
\frac{\partial \mathcal{R}}{\partial z_{\lambda}^{*}}= & -\frac{1}{4 \pi z_{\lambda}^{*}}-\frac{1}{4 \pi} \frac{\omega_{\alpha}\left(z_{\lambda}, z_{\lambda}^{*-1}\right)}{\omega\left(z_{\lambda}, z_{\lambda}^{*-1}\right)}\left(-\frac{1}{z_{\lambda}^{* 2}}\right) \\
& +\frac{1}{4 \pi} \frac{1}{\omega^{\prime *}\left(z_{\lambda}^{*}, z_{\lambda}^{*}\right)}\left(\omega_{\zeta}^{\prime *}\left(z_{\lambda}^{*}, z_{\lambda}^{*}\right)+\omega_{\alpha}^{\prime *}\left(z_{\lambda}^{*}, z_{\lambda}^{*}\right)\right)-\frac{1}{4 \pi} \frac{\omega_{\zeta}^{*}\left(z_{\lambda}^{*}, z_{\lambda}^{-1}\right)}{\omega^{*}\left(z_{\lambda}^{*}, z_{\lambda}^{-1}\right)} \\
= & -\frac{1}{4 \pi z_{\lambda}^{*}}+\frac{1}{4 \pi} \frac{\omega_{\alpha}\left(z_{\lambda}, z_{\lambda}^{*-1}\right)}{\omega\left(z_{\lambda}, z_{\lambda}^{*-1}\right)}\left(\frac{1}{z_{\lambda}^{* 2}}\right)-\frac{1}{4 \pi} \frac{\omega_{\zeta}^{*}\left(z_{\lambda}^{*}, z_{\lambda}^{-1}\right)}{\omega^{*}\left(z_{\lambda}^{*}, z_{\lambda}^{-1}\right)} .
\end{aligned}
$$

Note that the third term after the first equality vanishes due to (iv) of Lemma 1 . To reduce it further, we need the following lemma.

\section{Lemma 3}

$$
\frac{\omega_{\zeta}^{*}\left(z^{*}, z^{-1}\right)}{\omega^{*}\left(z^{*}, z^{-1}\right)}=-\frac{1}{\left(z^{*}\right)^{2}} \frac{\omega_{\alpha}\left(z, z^{*-1}\right)}{\omega\left(z, z^{*-1}\right)}+\frac{1}{z^{*}}
$$


Proof: It follows from (i) and (ii) of Lemma 1 that we obtain

$$
\omega^{*}\left(z^{*}, z^{-1}\right)=-\frac{z^{*}}{z} \omega\left(z^{*-1}, z\right)=\frac{z^{*}}{z} \omega\left(z, z^{*-1}\right) .
$$

Differentiating both sides of (i) of Lemma 1 with respect to $\zeta$, we have

$$
\frac{\partial}{\partial \zeta} \omega^{*}\left(\zeta^{-1}, \alpha^{-1}\right)=\omega_{\zeta}^{*}\left(\zeta^{-1}, \alpha^{-1}\right) \frac{-1}{\zeta^{2}}, \quad \frac{\partial}{\partial \zeta}\left(-\frac{1}{\zeta \alpha} \omega(\zeta, \alpha)\right)=\frac{1}{\zeta^{2} \alpha} \omega(\zeta, \alpha)-\frac{1}{\zeta \alpha} \omega_{\zeta}(\zeta, \alpha),
$$

which leads to

$$
\omega_{\zeta}^{*}\left(z^{*}, z^{-1}\right)=-\frac{1}{z} \omega\left(z^{*-1}, z\right)+\frac{1}{z^{*} z} \omega_{\zeta}\left(z^{*-1}, z\right) .
$$

Hence due to (i), (ii) and (v) of Lemma 1, we obtain

$$
\begin{aligned}
\frac{\omega_{\zeta}^{*}\left(z^{*}, z^{-1}\right)}{\omega^{*}\left(z^{*}, z^{-1}\right)} & =\frac{-\frac{1}{z} \omega\left(z^{*-1}, z\right)+\frac{1}{z^{*} z} \omega_{\zeta}\left(z^{*-1}, z\right)}{\frac{z^{*}}{z} \omega\left(z, z^{*-1}\right)} \\
& =\frac{\frac{1}{z} \omega\left(z, z^{*-1}\right)-\frac{1}{z^{*} z} \omega_{\alpha}\left(z, z^{*-1}\right)}{\frac{z^{*}}{z} \omega\left(z, z^{*-1}\right)}=\frac{1}{z^{*}}-\frac{\omega_{\alpha}\left(z, z^{*-1}\right)}{\left(z^{*}\right)^{2} \omega\left(z, z^{*-1}\right)} .
\end{aligned}
$$

Substituting (17) into (16), we finally obtain

$$
-2 \mathrm{i} \frac{\partial H_{R}}{\partial z_{\lambda}^{*}}=-\frac{\mathrm{i}}{2 \pi} \Gamma_{\lambda}^{2} \frac{\omega_{\zeta}^{*}\left(z_{\lambda}^{*}, z_{\lambda}^{-1}\right)}{\omega^{*}\left(z_{\lambda}^{*}, z_{\lambda}^{-1}\right)} .
$$

Consequently, the equation (5) is derived from (14) and (18).

\section{Motion of two point vortices in the circular domain}

\subsection{Reduction to a single point vortex in a semi-circle}

We consider the motion of two point vortices located at $z_{1}$ and $z_{2}$ in the circular domain $D_{\zeta}$ with the strengths $\Gamma_{1}=-\Gamma_{2}=1$. Then the equation (5) for the two point vortices is given by

$$
\begin{aligned}
\frac{d z_{1}}{d t} & =-\frac{\mathrm{i}}{2 \pi}\left(\frac{\omega_{\zeta}^{*}\left(z_{1}^{*}, z_{2}^{*}\right)}{\omega^{*}\left(z_{1}^{*}, z_{2}^{*}\right)}-\frac{\omega_{\zeta}^{*}\left(z_{1}^{*}, z_{2}^{-1}\right)}{\omega^{*}\left(z_{1}^{*}, z_{2}^{-1}\right)}\right)-\frac{\mathrm{i}}{2 \pi} \frac{\omega_{\zeta}^{*}\left(z_{1}^{*}, z_{1}^{-1}\right)}{\omega^{*}\left(z_{1}^{*}, z_{1}^{-1}\right)}, \\
\frac{d z_{2}}{d t} & =\frac{\mathrm{i}}{2 \pi}\left(\frac{\omega_{\zeta}^{*}\left(z_{2}^{*}, z_{1}^{*}\right)}{\omega^{*}\left(z_{2}^{*}, z_{1}^{*}\right)}-\frac{\omega_{\zeta}^{*}\left(z_{2}^{*}, z_{1}^{-1}\right)}{\omega^{*}\left(z_{2}^{*}, z_{1}^{-1}\right)}\right)+\frac{\mathrm{i}}{2 \pi} \frac{\omega_{\zeta}^{*}\left(z_{2}^{*}, z_{2}^{-1}\right)}{\omega^{*}\left(z_{2}^{*}, z_{2}^{-1}\right)} .
\end{aligned}
$$

When the domain is the unit circle or the doubly connected concentric annulus $\{\zeta|q<| \zeta \mid<1\}$, their motion is integrable, since the system admits an additional invariant quantity $I=\left|z_{1}\right|^{2}-\left|z_{2}\right|^{2}$, which corresponds to the invariance of the system with respect to the rotation around the origin. This is directly confirmed from the equations (19) and (20) as follows:

$$
\begin{aligned}
\frac{d I}{d t}= & 2 \operatorname{Re}\left(z_{1}^{*} \frac{d z_{1}}{d t}-z_{2}^{*} \frac{d z_{2}}{d t}\right) \\
= & -\frac{i}{\pi} \operatorname{Im}\left(z_{1}^{*} \frac{\omega_{\zeta}^{*}\left(z_{1}^{*}, z_{2}^{*}\right)}{\omega^{*}\left(z_{1}^{*}, z_{2}^{*}\right)}+z_{2}^{*} \frac{\omega_{\zeta}^{*}\left(z_{2}^{*}, z_{1}^{*}\right)}{\omega^{*}\left(z_{2}^{*}, z_{1}^{*}\right)}\right)+\frac{i}{\pi} \operatorname{Im}\left(z_{1}^{*} \frac{\omega_{\zeta}^{*}\left(z_{1}^{*}, z_{2}^{-1}\right)}{\omega^{*}\left(z_{1}^{*}, z_{2}^{-1}\right)}+z_{2}^{*} \frac{\omega_{\zeta}^{*}\left(z_{2}^{*}, z_{1}^{-1}\right)}{\omega^{*}\left(z_{2}^{*}, z_{1}^{-1}\right)}\right) \\
& -\frac{i}{\pi} \operatorname{Im}\left(z_{1}^{*} \frac{\omega_{\zeta}^{*}\left(z_{1}^{*}, z_{1}^{-1}\right)}{\omega^{*}\left(z_{1}^{*}, z_{1}^{-1}\right)}+z_{2}^{*} \frac{\omega_{\zeta}^{*}\left(z_{2}^{*}, z_{2}^{-1}\right)}{\omega^{*}\left(z_{2}^{*}, z_{2}^{-1}\right)}\right) .
\end{aligned}
$$


This vanishes if we have, for arbitrary $z, w \in \mathbb{C}$,

$$
\operatorname{Im}\left(z \frac{\omega_{\zeta}(z, w)}{\omega(z, w)}+w \frac{\omega_{\zeta}(w, z)}{\omega(w, z)}\right)=0, \quad\left(z \frac{\omega_{\zeta}\left(z, w^{*-1}\right)}{\omega\left(z, w^{*-1}\right)}\right)^{*}=w \frac{\omega_{\zeta}\left(w, z^{*-1}\right)}{\omega\left(w, z^{*-1}\right)} .
$$

For the unit circle, these conditions hold, since (8) satisfies

$$
z_{1} \frac{1}{z_{1}-z_{2}}+z_{2} \frac{1}{z_{2}-z_{1}}=1, \quad\left(z \frac{1}{z-w^{*-1}}\right)^{*}=\frac{z^{*}}{z^{*}-w^{-1}}=\frac{w}{w-z^{*-1}} .
$$

We also have the invariance of $I$ for the concentric annulus, since it follows from (9) that

$$
z_{1} \frac{q^{2 k}\left(z_{2}^{2}-z_{1}^{1}\right)}{\left(q^{2 k} z_{1}-z_{2}\right)\left(q^{2 k} z_{2}-z_{1}\right)} \frac{1}{z_{1}}+z_{2} \frac{q^{2 k}\left(z_{1}^{2}-z_{2}^{1}\right)}{\left(q^{2 k} z_{2}-z_{1}\right)\left(q^{2 k} z_{1}-z_{2}\right)} \frac{1}{z_{2}}=0,
$$

and

$$
\left(z \frac{q^{2 k}\left(w^{*-2}-z^{2}\right)}{\left(q^{2 k} z-w^{*-1}\right)\left(q^{2 k} w^{*-1}-z\right)} \frac{1}{z}\right)^{*}=\frac{q^{2 k}\left(1-z^{* 2} w^{2}\right)}{\left(q^{2 k} z^{*} w-1\right)\left(q^{2 k}-z^{*} w\right)}=w \frac{q^{2 k}\left(z^{*-2}-w^{2}\right)}{\left(q^{2 k} w-z^{*-1}\right)\left(q^{2 k} z^{*-1}-w\right)} \frac{1}{w} .
$$

Here, let us consider a circular domain that is symmetric with respect to the real axis. In other words, for arbitrary obstacle $C_{i}$, there exists an integer $j \in\{1, \ldots, M\}$ such that the obstacles $C_{j}$ and $C_{i}$ are symmetric with respect to the real axis, i.e., $\delta_{j}=\delta_{i}^{*}$ and $q_{j}=q_{i}$. Note that if $j=i$, the obstacle $C_{i}$ is symmetric with respect to the real axis. In terms of the maps in the Schottky group, it is equivalent to say that for arbitrary map $\theta_{i}(\zeta) \in \Theta^{\prime \prime}$, there exists a map $\theta_{j}(\zeta)$ such that $\theta_{i}^{*}(\zeta)=\theta_{j}(\zeta)$, since for any generating functions of the Schottky group, we have

$$
\left(\vartheta_{i}\left(\zeta^{*}\right)\right)^{*}=\left(\delta_{i}+\frac{q_{i}^{2} \zeta^{*}}{1-\delta_{i}^{*} \zeta^{*}}\right)^{*}=\delta_{i}^{*}+\frac{q_{i}^{2} \zeta}{1-\delta_{i} \zeta}=\delta_{j}+\frac{q_{j}^{2} \zeta}{1-\delta_{j}^{*} \zeta}=\vartheta_{j}(\zeta) .
$$

Then, for arbitrary $\zeta$ and $\alpha \in D_{\zeta}$, we obtain

$$
\begin{aligned}
\frac{\omega_{\zeta}^{*}(\zeta, \alpha)}{\omega^{*}(\zeta, \alpha)} & =\left(\frac{\omega_{\zeta}\left(\zeta^{*}, \alpha^{*}\right)}{\omega\left(\zeta^{*}, \alpha^{*}\right)}\right)^{*} \\
& =\left(\frac{1}{\zeta^{*}-\alpha^{*}}+\sum_{\theta_{i} \in \Theta^{\prime \prime}}\left\{\frac{\theta_{i}^{\prime}\left(\zeta^{*}\right)\left(\alpha^{*}-\zeta^{*}\right)}{\left(\theta_{i}\left(\zeta^{*}\right)-\alpha^{*}\right)\left(\theta_{i}\left(\zeta^{*}\right)-\zeta^{*}\right)}+\frac{\theta_{i}\left(\alpha^{*}\right)-\theta_{i}\left(\zeta^{*}\right)}{\left(\theta_{i}\left(\alpha^{*}\right)-\zeta^{*}\right)\left(\theta_{i}\left(\zeta^{*}\right)-\zeta^{*}\right)}\right\}\right)^{*} \\
& =\frac{1}{\zeta-\alpha}+\sum_{\theta_{i} \in \Theta^{\prime \prime}}\left\{\frac{\theta_{i}^{\prime *}(\zeta)(\alpha-\zeta)}{\left(\theta_{i}^{*}(\zeta)-\alpha\right)\left(\theta_{i}^{*}(\zeta)-\zeta\right)}+\frac{\theta_{i}^{*}(\alpha)-\theta_{i}^{*}(\zeta)}{\left(\theta_{i}^{*}(\alpha)-\zeta\right)\left(\theta_{i}^{*}(\zeta)-\zeta\right)}\right\} \\
& =\frac{1}{\zeta-\alpha}+\sum_{\theta_{j} \in \Theta^{\prime \prime}}\left\{\frac{\theta_{j}^{\prime}(\zeta)(\alpha-\zeta)}{\left(\theta_{j}(\zeta)-\alpha\right)\left(\theta_{j}(\zeta)-\zeta\right)}+\frac{\theta_{j}(\alpha)-\theta_{j}(\zeta)}{\left(\theta_{j}(\alpha)-\zeta\right)\left(\theta_{j}(\zeta)-\zeta\right)}\right\}=\frac{\omega_{\zeta}(\zeta, \alpha)}{\omega(\zeta, \alpha)} .(21)
\end{aligned}
$$

Suppose that the initial location of the two point vortices is $z_{1}=z_{2}^{*}$. Then due to (21) we have

$$
\begin{aligned}
\frac{d}{d t}\left(z_{1}-z_{2}^{*}\right)= & -\frac{\mathrm{i}}{2 \pi} \frac{\omega_{\zeta}^{*}\left(z_{1}^{*}, z_{2}^{*}\right)}{\omega^{*}\left(z_{1}^{*}, z_{2}^{*}\right)}+\frac{\mathrm{i}}{2 \pi} \frac{\omega_{\zeta}^{*}\left(z_{1}^{*}, z_{2}^{-1}\right)}{\omega^{*}\left(z_{1}^{*}, z_{2}^{-1}\right)}-\frac{\mathrm{i}}{2 \pi} \frac{\omega_{\zeta}^{*}\left(z_{1}^{*}, z_{1}^{-1}\right)}{\omega^{*}\left(z_{1}^{*}, z_{1}^{-1}\right)} \\
& -\left(\frac{\mathrm{i}}{2 \pi} \frac{\omega_{\zeta}^{*}\left(z_{2}^{*}, z_{1}^{*}\right)}{\omega^{*}\left(z_{2}^{*}, z_{1}^{*}\right)}-\frac{\mathrm{i}}{2 \pi} \frac{\omega_{\zeta}^{*}\left(z_{2}^{*}, z_{1}^{-1}\right)}{\omega^{*}\left(z_{2}^{*}, z_{1}^{-1}\right)}+\frac{\mathrm{i}}{2 \pi} \frac{\omega_{\zeta}^{*}\left(z_{2}^{*}, z_{2}^{-1}\right)}{\omega^{*}\left(z_{2}^{*}, z_{2}^{-1}\right)}\right)^{*} \\
= & -\frac{\mathrm{i}}{2 \pi} \frac{\omega_{\zeta}^{*}\left(z_{1}^{*}, z_{1}\right)}{\omega^{*}\left(z_{1}^{*}, z_{1}\right)}+\frac{\mathrm{i}}{2 \pi} \frac{\omega_{\zeta}^{*}\left(z_{1}^{*}, z_{1}^{*-1}\right)}{\omega^{*}\left(z_{1}^{*}, z_{1}^{*-1}\right)}-\frac{\mathrm{i}}{2 \pi} \frac{\omega_{\zeta}^{*}\left(z_{1}^{*}, z_{1}^{-1}\right)}{\omega^{*}\left(z_{1}^{*}, z_{1}^{-1}\right)} \\
& +\frac{\mathrm{i}}{2 \pi} \frac{\omega_{\zeta}\left(z_{1}^{*}, z_{1}\right)}{\omega\left(z_{1}^{*}, z_{1}\right)}-\frac{\mathrm{i}}{2 \pi} \frac{\omega_{\zeta}\left(z_{1}^{*}, z_{1}^{*-1}\right)}{\omega\left(z_{1}^{*}, z_{1}^{*-1}\right)}+\frac{\mathrm{i}}{2 \pi} \frac{\omega_{\zeta}\left(z_{1}^{*}, z_{1}^{-1}\right)}{\omega\left(z_{1}^{*}, z_{1}^{-1}\right)}=0 .
\end{aligned}
$$


Thus we prove the following theorem.

Theorem 3 Let the circular domain $D_{\zeta}$ be symmetric with respect to the real axis. Suppose also that the initial configuration of the two point vortices with $\Gamma_{1}=-\Gamma_{2}=1$ satisfies $z_{1}(0)=z_{2}^{*}(0)$. Then we have $z_{1}(t)=z_{2}^{*}(t)$ for $t \geq 0$.

Owing to this theorem, the orbits of the two point vortices in the circular domain are symmetric with respect to the real axis, which are observed by plotting the contour lines of the Hamiltonian with $z_{1}=z_{2}^{*}$, i.e.,

$$
H\left(z_{1}, z_{1}^{*}\right)=G\left(z_{1} ; z_{1}^{*}\right)-\frac{1}{2} \mathcal{R}\left(z_{1} ; z_{1}^{*}\right)-\frac{1}{2} \mathcal{R}\left(z_{1}^{*} ; z_{1}\right) .
$$

Hence it is possible to investigate the motion of the two point vortices by plotting the contour lines of the Hamiltonian (22).

Finally let us mention how to compute the Schottky-Klein prime function $\omega(\zeta, \alpha)$ numerically. Since it is unable to calculate the infinite product in (4), we have to truncate it up to a finite number of the Möbius maps in $\Theta^{\prime \prime}$. As in [3], we introduce the level of all possible compositions of the generating maps $\vartheta_{i}(\zeta)$ and $\vartheta_{i}^{-1}(\zeta)$. The level-zero map is the identity map. The level-one maps contain all generating maps $\vartheta_{i}(\zeta)$ and their inverse $\vartheta_{i}^{-1}(\zeta)$ for $i=1, \ldots, M$. All compositions of any two of the level-one maps that cannot be reduced to the identity map constitute the level-two maps. We can define recursively the level-three maps that consist of all possible combinations of three of the level-one maps and that are not reduced to a lower-level map. In the present paper, we have truncated the infinite product (4) up to the level-three maps. The truncated finite product approximates the Schottky-Klein prime function accurately if the obstacles $C_{i}$ for $i=1, \ldots, M$ are well-separated as is confirmed in [3]. As for the infinite summation (6) in Proposition 1, we similarly truncate it up to the level-three maps.

\subsection{Motion of a point vortex in multiply connected semi-circles}

\subsubsection{Two obstacles}

Due to the reflectional symmetry, the two obstacles $C_{1}$ and $C_{2}$ are symmetric with respect to the real axis. Namely, the centers and the radii of the two obstacles are given by

$$
C_{1}: \delta_{1}=a+b \mathrm{i}, \quad q_{1}=r, \quad C_{2}: \delta_{2}=a-b \mathrm{i}, \quad q_{2}=r,
$$

for $a \in(-1,1)$ and $b>0$. The radius $r$ satisfies $0<r<\min \left(b, 1-\sqrt{a^{2}+b^{2}}\right)$, since the obstacle $C_{1}$ must be contained in the upper semi-circle.

Figure 1 shows the contour plots of the Hamiltonian (22) for $a=0, r=0.05$ and various $b$ in (23). Saddle and center points of the contour plot correspond to unstable and neutrally stable fixed configurations of the two point vortices respectively. The contour plots are symmetric with respect to the real axis, which means that when the point vortex with the positive unit strength goes along a contour line in the upper semi-circle, the other point vortex with the negative unit strength always moves along the contour line in the lower semi-circle that is symmetric with respect to the real axis. Thus it is sufficient for us to focus on the contour lines in the upper semi-circle. When $b=0.2$ in Figure 1(a), there are two fixed configurations, a center point above the obstacle $C_{1}$ and a saddle point between the obstacle and the real axis. The saddle point is connected by homoclinic orbits. For $b=0.4$ in Figure 1(b), while the saddle point with the homoclinic connections below the obstacle remains, a new saddle point appears above the obstacle. The new saddle point is connected by homoclinic orbits that surround two center points. At $b=0.4885$ 


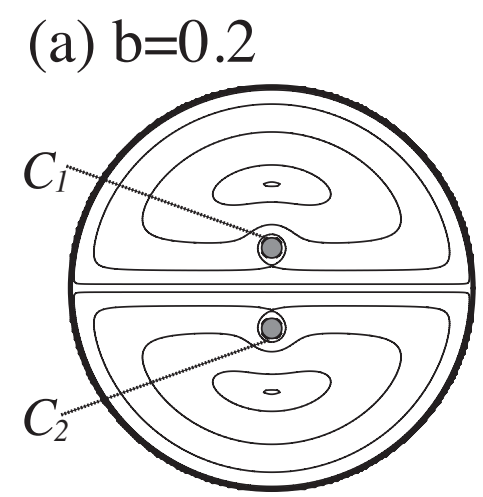

(d) $b=0.6$

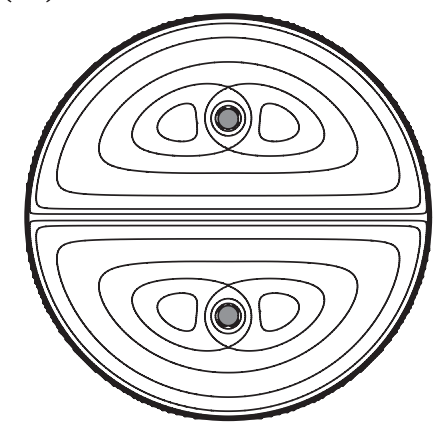

(b) $b=0.4$

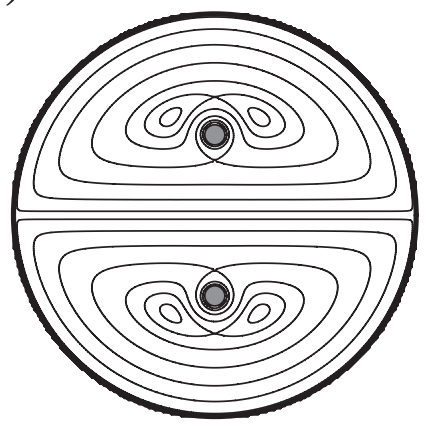

(e) $b=0.8$

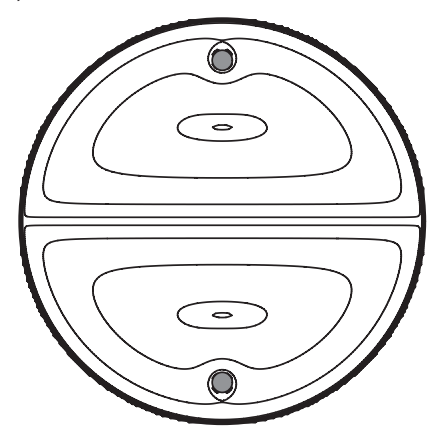

(c) $b=0.4885$

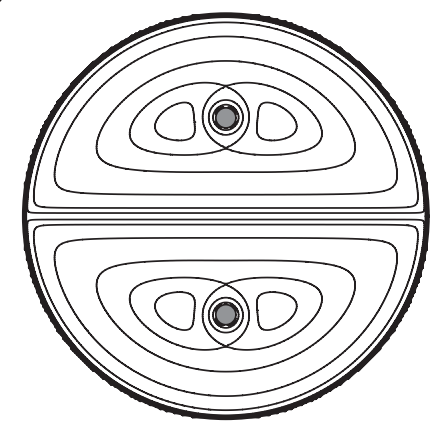

Figure 1: Contour plots of the Hamiltonian (22) in the circular domains with two obstacles that are symmetric with respect to the real axis. In (23), we change $b$ with fixed $a=0$ and $r=0.05$.

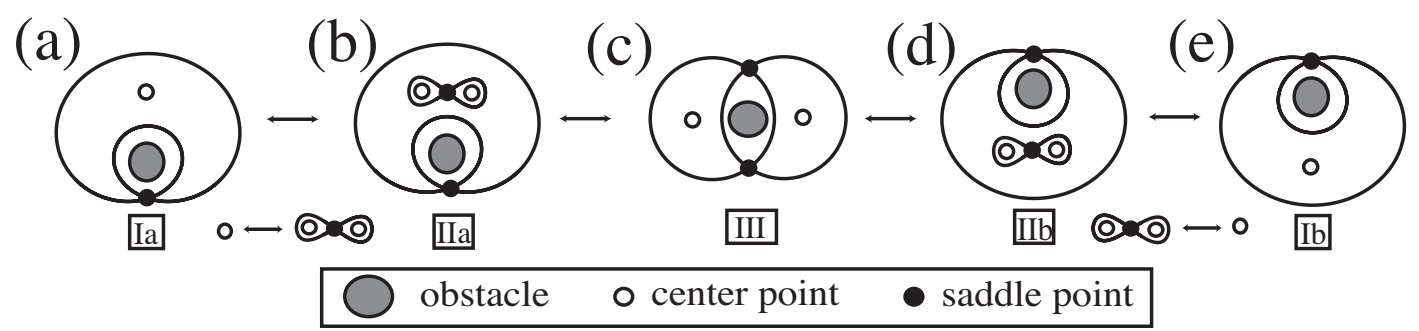

Figure 2: Topological patterns of the contour lines in the upper semi-circles corresponding to the contour plots given in Figure 1 


\begin{tabular}{|c|c|c|c|c|c|c|c|c|c|c|c|c|c|c|c|}
\hline$a=0.3$ & \multicolumn{8}{|c|}{$b$} & & \multirow{2}{*}{\multicolumn{6}{|c|}{$b$}} \\
\hline$r$ & 0.1 & 0.2 & 0.3 & 0.4 & 0.5 & 06 & 07 & 08 & $a=0.7$ & & & & & & \\
\hline 0.03 & Ia & $\mathrm{Ia}$ & $\mathrm{Ia}$ & $\mathrm{Ia}$ & $\mathrm{Ib}$ & $\overline{\mathrm{Ib}}$ & $\mathrm{Ih}$ & 0.0 & $r$ & 0.1 & 0.2 & 0.3 & 0.4 & 0.5 & 0.6 \\
\hline 0.1 & & Ia & $\mathrm{Ia}$ & IIa & IJb & Jb & $\mathrm{Ib}$ & Ib & 0.05 & Ia & $\mathrm{Ia}$ & $\mathrm{Ib}$ & $\mathrm{Ib}$ & $\mathrm{Ib}$ & $\mathrm{Ib}$ \\
\hline 0.15 & & $\mathrm{Ia}$ & IIa & Ша & $\mathrm{IIb}$ & Ith & 10 & & 0.1 & & IIa & IIb & $\mathrm{Ib}$ & $\mathrm{Ib}$ & \\
\hline 0.2 & & & $\mathrm{IIa}$ & IIa & Uh & 110 & 10 & & 0.15 & & & IIa & IIb & IIb & \\
\hline 0.3 & & & & IIa & $\mathrm{IIb}$ & IID & & & 0.2 & & & IIa & & & \\
\hline
\end{tabular}

Table 1: Classification of topological patterns of the contour lines of the Hamiltonian (22) in the upper semi-circle for $a=0.3$ and $a=0.7$.

in Figure 1(c), the homoclinic orbits with respect to the two saddle points coincide, and then two saddles with homoclinic connections appear again for $b=0.6$ in Figure 1(d). Finally, when the island approaches the outer boundary for $b=0.8$ in Figure 1(e), the saddle point below the obstacle $C_{1}$ changes to a center point.

The motion of the two point vortices changes largely in the neighborhood of the saddle points of the contour plot. For instance, in Figure 1(a), when we set the point vortex below the saddle point at the initial moment, it goes along the real axis and the boundary of the unit circle. On the other hand, it rotates around the obstacle $C_{1}$ when it is initially set above the saddle. Thus, in order to characterize the motion of the single point vortex in the upper semi-circle, we pay attention to the topological structure that consists of the saddle/center points, their heteroclinic/homoclinic orbits in the contour lines of the Hamiltonian (22) and the obstacle $C_{1}$. In Figure 2, we show the topological patterns corresponding to the contour plots from Figure 1(a) to (e). The topological patterns for $b=0.2$ and 0.8 , and those for $b=0.4$ and 0.6 are the same topologically. Hence, the topological patterns are referred to as Ia for $b=0.2$, IIa for $b=0.4$, III for $b=0.4885$, IIb for $b=0.6$ and Ib for $b=0.8$. Figure 2 also illustrates how the topological patterns change with respect to $b$. The changes from the pattern Ia to IIa, and from the pattern IIb to Ib are due to a pitchfork bifurcation from the neutrally stable fixed configuration to the unstable one with the homoclinic connections, which gives rise to the two neutrally stable fixed configurations. The transition between the patterns IIa and IIb occurs due to the reconnection of the homoclinic orbits through the degenerate pattern III. Table 1 gives the classification of the topological patterns of the contour lines for the other cases $a=0.3$ and 0.7 with various $b$ and $r$, in which we typically observe the patterns Ia, Ib, IIa and IIb. The degenerate pattern III also appears for the parameters between the patterns IIa and IIb. We have examined the topological patterns for the other values of $a$, but we find no other pattern except them.

\subsubsection{Three obstacles}

Since the circular domain with three obstacles has the reflectional symmetry, the center of one obstacle is on the real axis. Thus the centers and the radii of the three obstacles are given by

$$
C_{1}: \delta_{1}=a+b \mathrm{i}, \quad q_{1}=r_{1}, \quad C_{2}: \delta_{2}=a-b \mathrm{i}, \quad q_{2}=r_{1}, \quad C_{3}: \delta_{3}=c, \quad q_{3}=r_{2},
$$

for $a$ and $c \in(-1,1)$, and the positive $b, r_{1}$ and $r_{2}$. Figure 3 shows the contour plots of the Hamiltonian (22) in the circular domains with the three obstacles (24) for $a=0, b=0.8, r_{1}=0.05$ and $c=0$. We change the radius $r_{2}$ of the obstacle $C_{3}$. For small $r_{2}$, the topological pattern is equivalent to the pattern Ib in Figure 2. As $r_{2}$ gets larger, the topological pattern changes to $\mathrm{IIb}$ and IIa. Figure 4 gives the other contour plots of the Hamiltonian (22) for $a=0, b=0.6$, 
(a) $r_{2}=0.05$

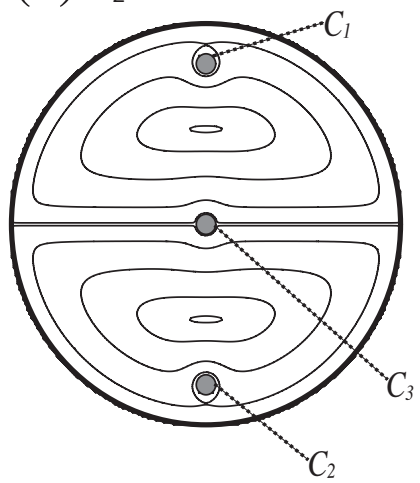

(b) $\mathrm{r}_{2}=0.5$

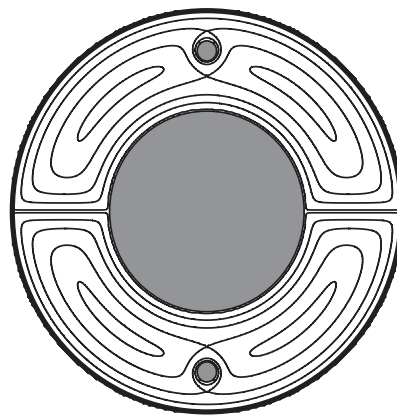

(c) $r_{2}=0.7$

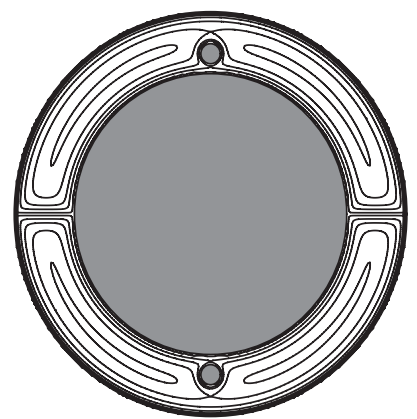

Figure 3: Contour plots of the Hamiltonian (22) in the symmetric circular domains with the three obstacles (24) for $a=0, b=0.8, r_{1}=0.05$ and $c=0$. We change the radius $r_{2}$ of the obstacle $C_{3}$.
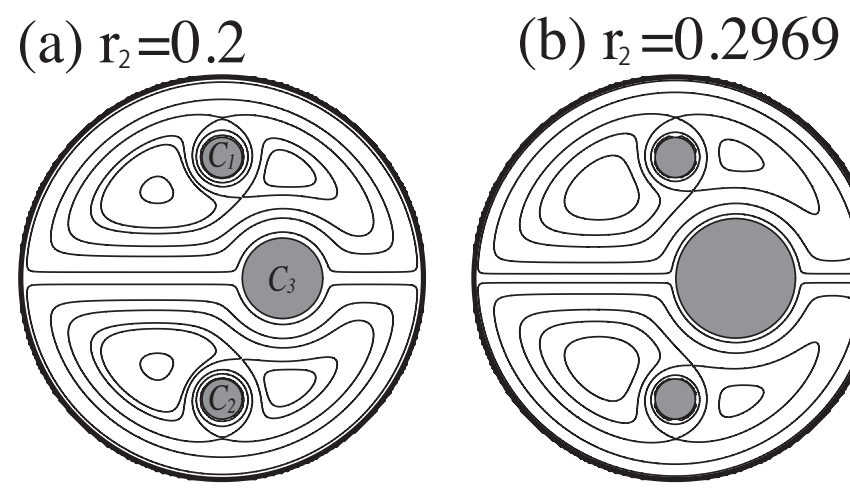

(c) $r_{2}=0.4$
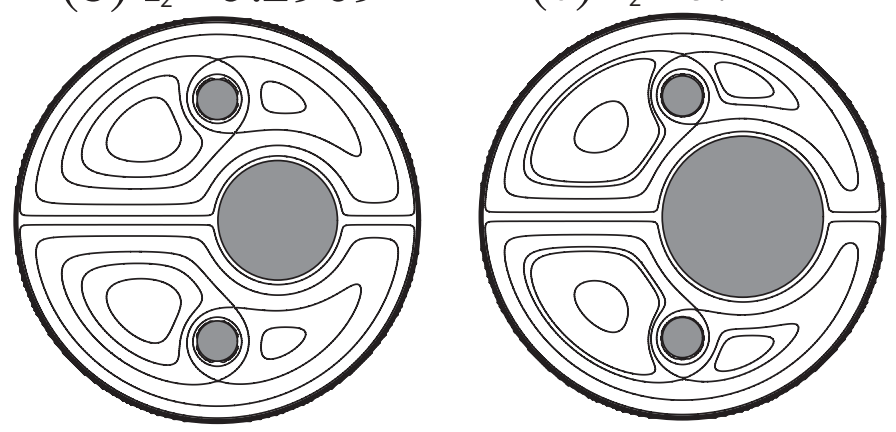

Figure 4: Contour plots of the Hamiltonian (22) in the symmetric circular domains with the three obstacles (24) for $a=0, b=0.6, r_{1}=0.1$ and $c=0.3$. We change the radius $r_{2}$ of the obstacle $C_{3}$.

$r_{1}=0.1$ and $c=0.3$, which clearly demonstrates the transition of the contour pattern from IIb to IIa through the degenerate pattern III at $r_{2}=0.2969$. We have checked the other configurations of the three obstacles, but we are unable to find any other topological patterns except those in Figure 2. Hence, we could say that the topological patterns observed here are the same as those in $\S 3.2 .1$.

\subsubsection{Four obstacles}

We need to consider many parameters in order to determine the locations of four islands. Hence, in this section, we deal with a special configuration of the four circular obstacles of the same radius. The centers of the obstacles are represented by

$$
C_{1}: \delta_{1}=a+b \mathrm{i}, \quad C_{2}: \delta_{2}=a-b \mathrm{i}, \quad C_{3}: \delta_{3}=-a+b \mathrm{i}, \quad C_{4}: \delta_{4}=-a-b \mathrm{i}, \quad a, b>0 .
$$

The radii of the four obstacles are fixed to 0.1 . 

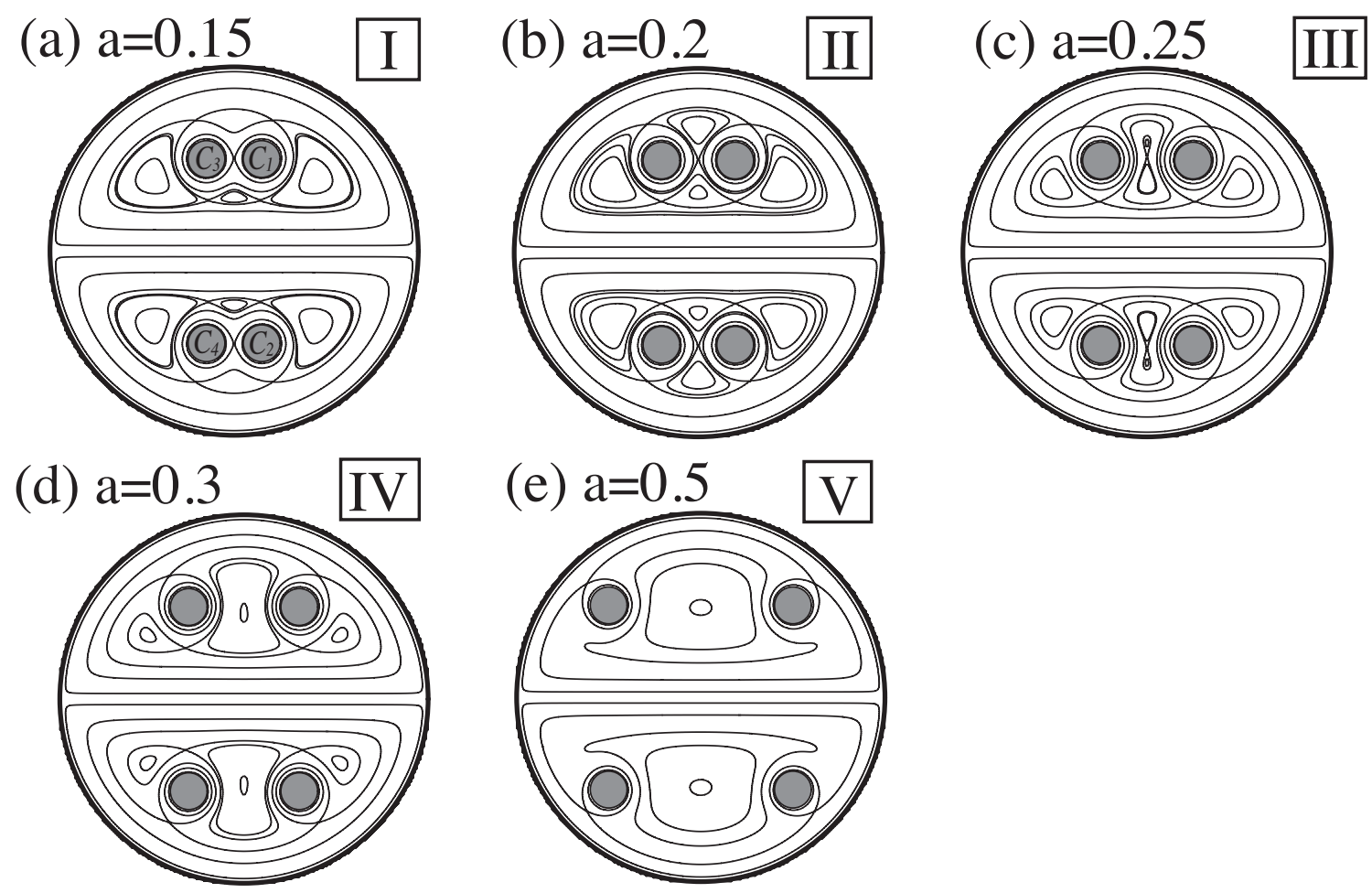

Figure 5: Contour plots of the Hamiltonian (22) in the symmetric circular domains with the four obstacles of radius 0.1 at (25), in which we change $a$ with fixed $b=0.5$.

First, we fix $b=0.5$ and change $a$. Figure 5 shows the contour plots of the Hamiltonian (22). When $a=0.15$ in Figure 5(a), in which the two obstacles $C_{1}$ and $C_{3}$ are close, there exists a saddle point between the two obstacles with homoclinic connections. In addition, we have two saddles and three centers below the obstacles, and two saddles and one center above the obstacles. Each saddle point is connected by homoclinic and heteroclinic orbits. For $a=0.2$ in Figure 5(b) and $a=0.25$ in Figure 5(c), the number of the saddle and center points remains the same as we have in Figure 5(a), but their global topological patterns of the homoclinic and the heteroclinic orbits are different. As the parameter $a$ increases, the saddle point between the two obstacles changes to a center point as in Figure 5(d) for $a=0.3$. The two saddles and the three centers below the obstacles collapse to a center in Figure 5(e) for $a=0.5$. We obtain the five topological patterns, which are referred to as I for $a=0.15$, II for $a=0.2$, III for $a=0.25$, IV for $a=0.3$ and V for $a=0.5$ respectively.

The transitions between the patterns I, II and III occur not due to the change of stability of the fixed configuration, but due to reconnection of the homoclinic and heteroclinic orbits through the degenerate cases for $a=0.1935$ in Figure 6(a) and for $a=0.21269$ in Figure 6(b). The subcritical pitchfork bifurcation from saddle to center results in the change of the topological pattern from III to IV. The transition between the patterns IV and V arises due to a degenerate pinching of the contour lines of the Hamiltonian around the center point below the obstacles as shown in Figure $7(\mathrm{a})$.

Next, we change $b$ with $a=0.15$. Figure 8 shows the contour plots of the Hamiltonian (22). When the two obstacles $C_{1}$ and $C_{3}$ are close to the real axis for $b=0.15$ in Figure 8(a), we have two saddle points with heteroclinic and homoclinic connections between the obstacles and 

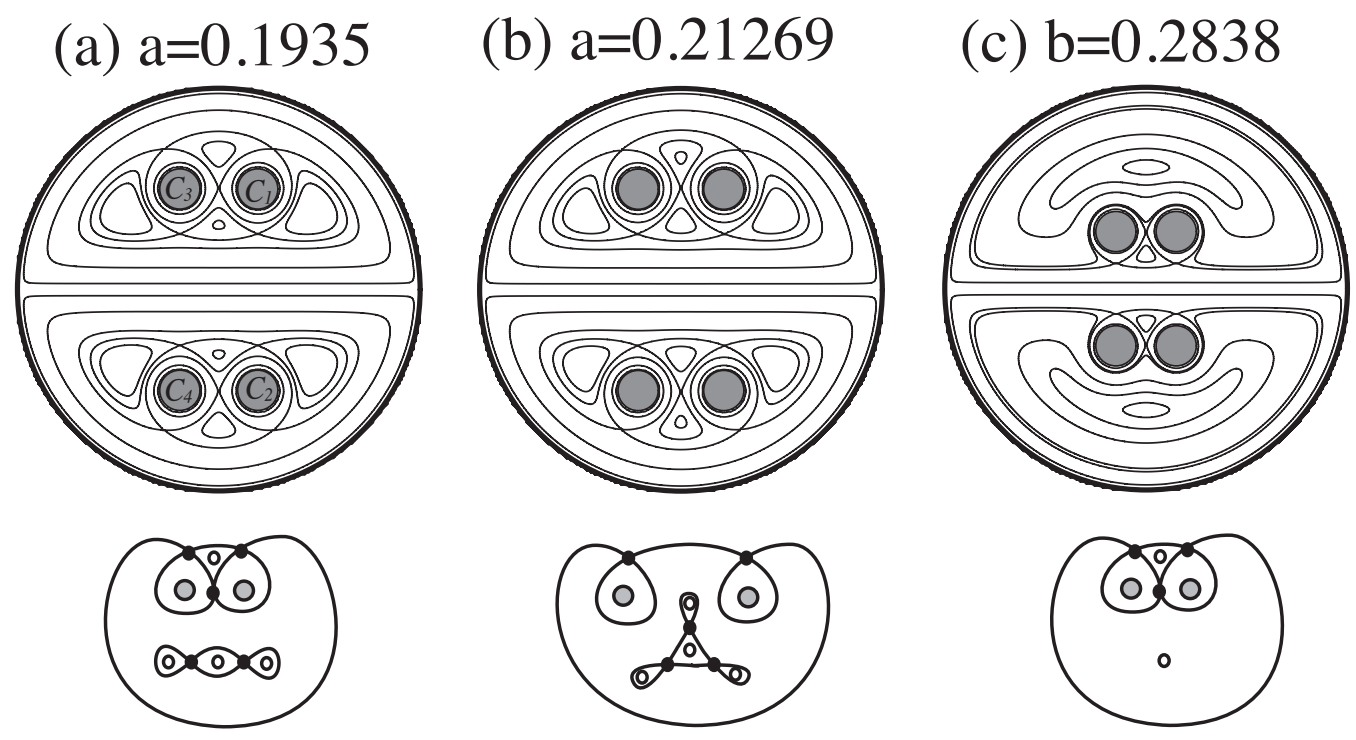

Figure 6: Contour plots of the Hamiltonian (22) and their corresponding topological patterns of the contour lines in the upper semi-circles for (a) $a=0.1935, b=0.5$, (b) $a=0.21269, b=0.5$ and (c) $a=0.15, b=0.2838$ in (25). They are degenerate cases through which the reconnections of the homoclinic and heteroclinic orbits of the topological patterns occur.

(a)
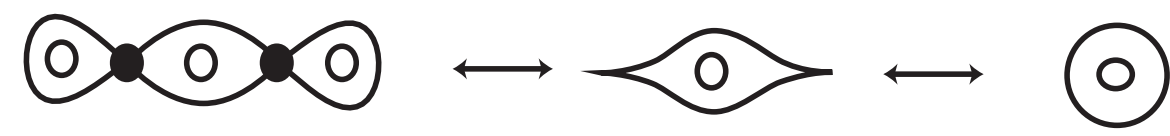

(b)
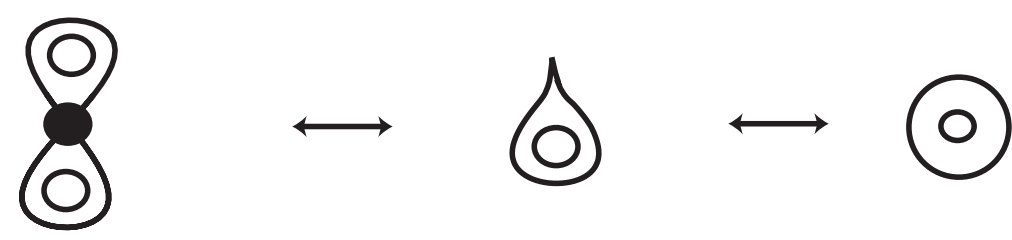

Figure 7: Schematic pictures for degenerate pinching bifurcations of the contour lines of the Hamiltonian around the center points, which generate new saddle/center points and homoclinic/heteroclinic connections. 

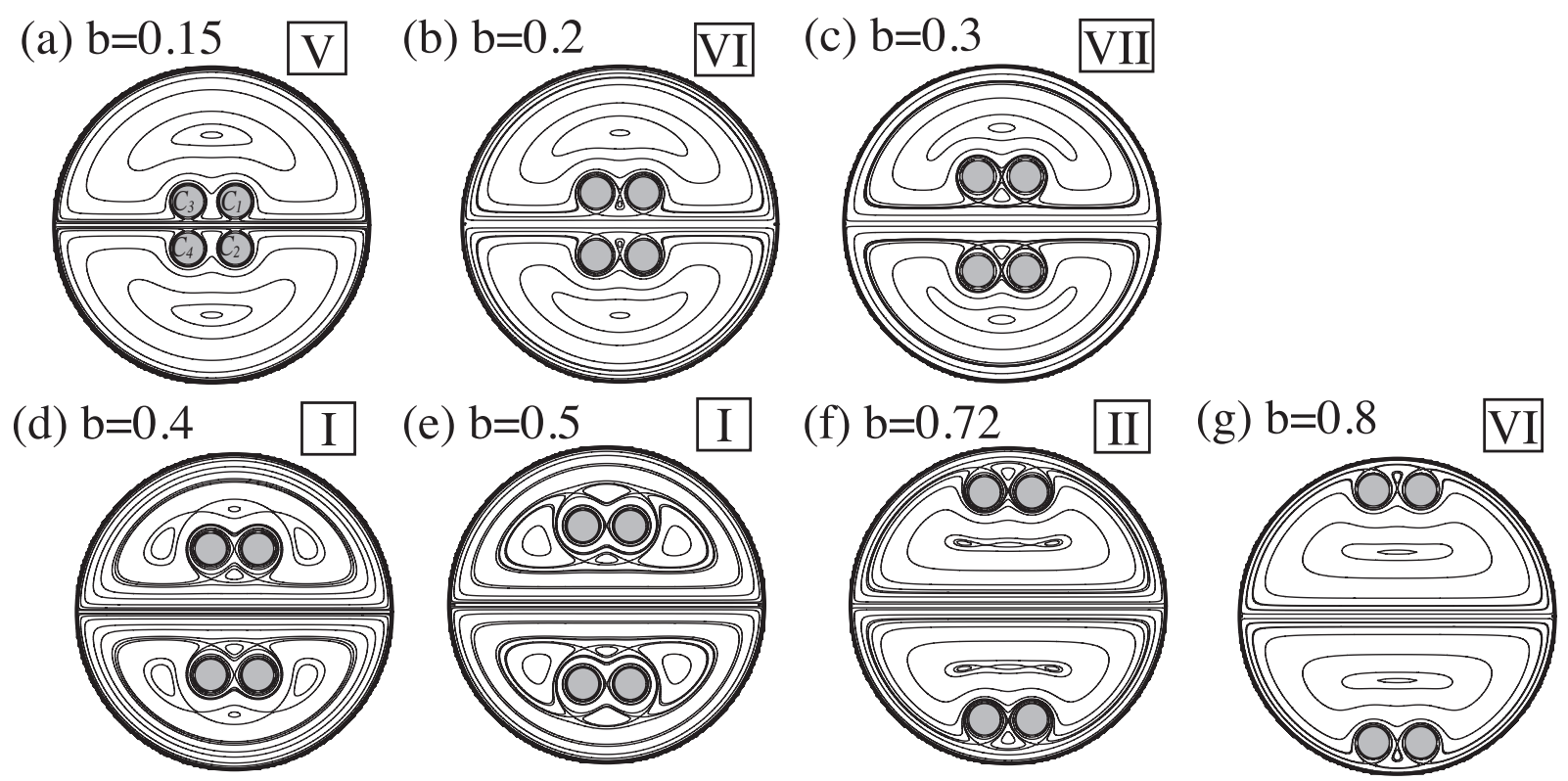

Figure 8: Contour plots of the Hamiltonian (22) in the symmetric circular domains with the four obstacles of radius 0.1 at (25), in which we change $b$ with $a=0.15$.

the real axis, and a center point above them. The pattern is topologically equivalent to the pattern $\mathrm{V}$ for Figure 5(e). Another degenerate pinching like Figure 7(b) generates a saddle point with homoclinic connections for $b=0.2$ in Figure $8(\mathrm{~b})$, which arises a new pattern VI. A global reconnection of the heteroclinic and homoclinic orbits occurs from Figure 8(b) to Figure 8(c) for $b=0.3$, which is identified as a new pattern VII. The degenerate topological pattern between the patterns VI and VII for $b=0.2838$ is given in Figure 6(c). The pinching bifurcation of Figure 7(a) around the center above the obstacles results in the transition from the pattern VII to I for $b=0.4$ and 0.5 in Figure 8(d) and (e). Then the global reconnection from the pattern I to II is observed for $b=0.72$ in Figure $8(\mathrm{f})$ through the degenerate pattern like in Figure 6(a). The transition from the pattern II to VI is due to the pinching bifurcation of Figure 7(b) around the center point below the two obstacles. These seven topological patterns I to VII are typically observed for the other configurations of the obstacles (25). Table 2 gives the classification of the topological patterns of the contour lines for $a=0.2,0.25$ and 0.3 with various $b$, in which we observe the patterns II, III, IV and V. For $a=0.2$, a new transition from the pattern II to IV is observed due to the degenerate pinching of Figure 7(b). We summarize the transitions and the bifurcations of the topological patterns of the contour lines in the upper semi-circle in Figure 9. Let us note that we are unable to find any other generic patterns except the seven patterns although we have examined the patterns for the other possible configurations of the four obstacles.

\subsection{Comparison with the motion of a single point vortices in circles}

As is shown in [4], since the motion of a single point vortex in any circular domain is integrable, its orbit corresponds to a contour line of the following Hamiltonian

$$
H^{(s)}\left(z_{1}\right)=-\frac{1}{2} \mathcal{R}\left(z_{1} ; z_{1}^{*}\right)
$$




\begin{tabular}{|c|cccccc|}
\hline & $\mathrm{b}=0.3$ & $\mathrm{~b}=0.34$ & $\mathrm{~b}=0.4$ & $\mathrm{~b}=0.5$ & $\mathrm{~b}=0.65$ & $\mathrm{~b}=0.7$ \\
\hline$a=0.2$ & $\mathrm{~V}$ & IV & II & II & IV & $\mathrm{V}$ \\
\hline \hline & $\mathrm{b}=0.2$ & $\mathrm{~b}=0.4$ & $\mathrm{~b}=0.47$ & $\mathrm{~b}=0.5$ & $\mathrm{~b}=0.6$ & $\mathrm{~b}=0.8$ \\
\hline$a=0.25$ & $\mathrm{~V}$ & IV & III & III & IV & $\mathrm{V}$ \\
\hline \hline & $\mathrm{b}=0.2$ & $\mathrm{~b}=0.4$ & $\mathrm{~b}=0.5$ & $\mathrm{~b}=0.65$ & & \\
\hline$a=0.5$ & $\mathrm{~V}$ & IV & IV & V & & \\
\hline
\end{tabular}

Table 2: Classification of the topological patterns of the contour lines of the Hamiltonian (22) in the upper semi-circles for $a=0.2, a=0.25$ and $a=0.3$ in (25).

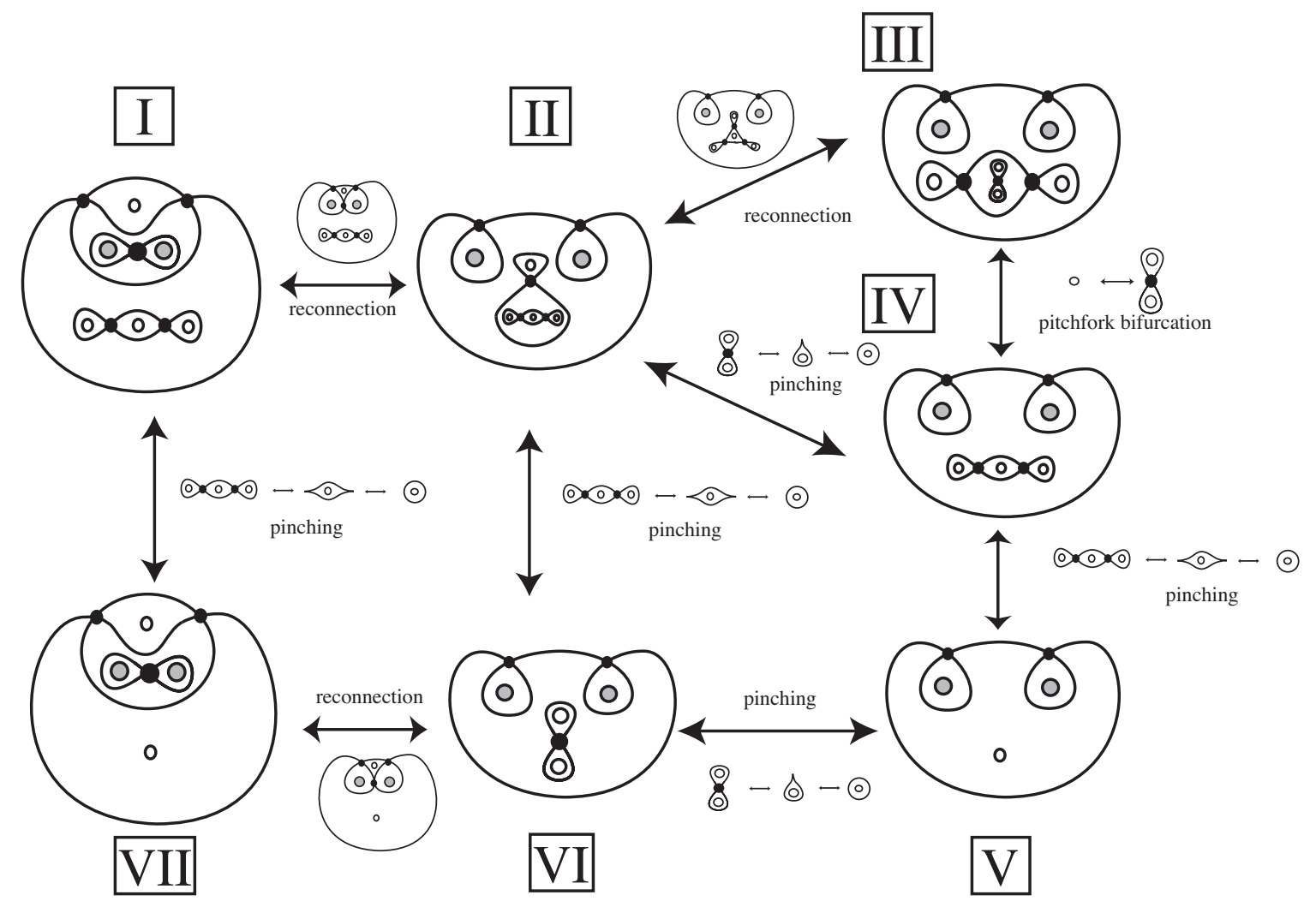

Figure 9: Transition diagram of the topological patterns of the contour lines of the Hamiltonian in the upper semi-circles found in Figures 5, 6 and 8. 


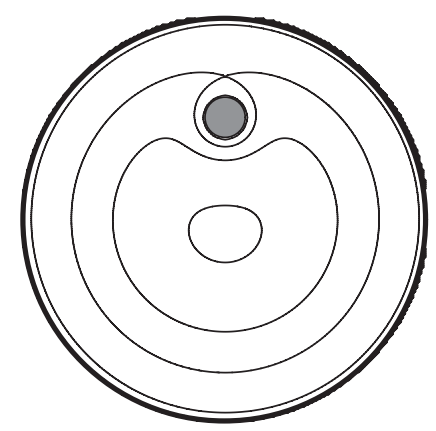

Figure 10: Contour plot of the Hamiltonian (26) for the single point vortex in the doubly connected circular domain with one obstacle of radius 0.1 , whose center is located at $0.5 \mathrm{i}$. Its corresponding topological pattern is equivalent to the pattern Ib in Figure 2.

which is obtained by taking $N=1$ in (1). We investigate the topological pattern of the contour lines of (26) for circular domains with the same connectivity as the semi-circles considered in $\S 3.2$.

We first deal with the motion of a point vortex in a doubly connected circular domain with one obstacle, which we compare with the results in $\S \S 3.2 .1$ and 3.2.2. Due to the rotational symmetry of the unit circle, the center of the obstacle is given by $\delta_{1}=b$ for $b>0$ without loss of generality. Figure 10 shows the contour plot of $H^{(s)}$ for $b=0.5$. The radius of the obstacle is 0.1 . We have one saddle point with homoclinic connections and one center point, whose corresponding topological pattern is equivalent to the pattern Ib in Figure 2. For the other value of $b$ and the radius of the obstacle, the only topological pattern we observe is the pattern Ib.

Next, we consider the motion of a point vortex in a triply connected circular domain. Since the configuration of the four obstacles $(25)$ in $§ 3.2 .3$ has the special symmetry, for the sake of comparison, we assume that the centers of two obstacles in the circular domain are located at

$$
C_{1}: \delta_{1}=a+b \mathrm{i}, \quad C_{2}: \delta_{2}=-a+b \mathrm{i}, \quad a>0, b \geq 0 .
$$

and the radii of the two obstacles are both 0.1. Figure 11 shows the contour plots of the Hamiltonian (26) with $b=0$ and various $a$. The topological patterns for $a=0.2, a=0.3$ and $a=0.5$ are equivalent to the patterns VII, VI and V in Figure 9 respectively. The global reconnection of the homoclinic orbits occurs between the patterns VII and VI through the degenerate pattern like Figure 6(c). The saddle point between the obstacles in Figure 11(b) becomes the center point in Figure 11(c) due to the pitchfork bifurcation. The transition and the bifurcation between the three patterns are observed for the other parameters $a$ and $b$ as shown in Table 3.

These results indicate that the possible topological patterns of the contour lines for the motion of the single point vortex in the circular domain are given as a part of those observed in the semi-circle with the same connectivity. Hence, we can observe a wide variety of the topological patterns of the contour lines and the transitions and the bifurcations between them in the motion of the single point vortex in the multiply connected semi-circle compared to those in the multiply connected circle.

\subsection{Nonintegrable motion of the two point vortices without the symmetry}

When either the circular domain or the initial configuration of the two point vortices is not symmetric with respect to the real axis, the system is no longer integrable. Here we give some numerical samples of the motion of the two point vortices without the reflectional symmetry. The 

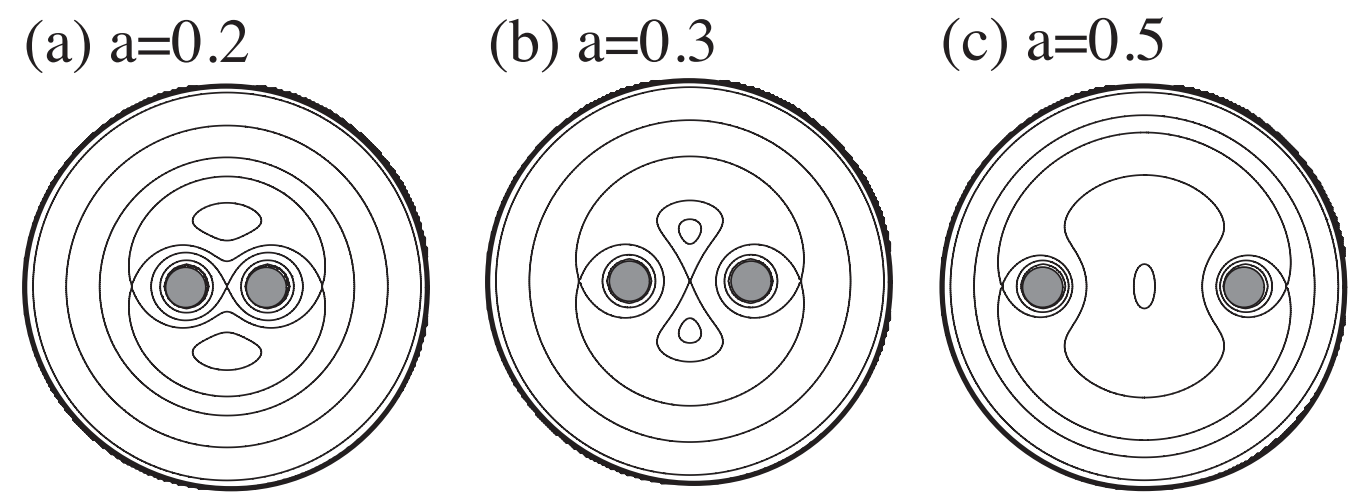

Figure 11: Contour plots of the Hamiltonian (26) for the single point vortex in the triply connected circular domains with the two obstacles at (27) with $b=0$ and various $a$, whose topological patterns for $a=0.2,0.3$ and 0.5 are equivalent to (a) the pattern VII, (b) VI and (c) V in Figure 9 respectively.

\begin{tabular}{|c|cccccc|}
\hline & $b=0.1$ & $b=0.2$ & $b=0.3$ & $b=0.4$ & $b=0.5$ & $b=0.6$ \\
\hline$a=0.2$ & VII & VI & VI & VI & VI & V \\
$a=0.3$ & VI & V & V & V & V & V \\
$a=0.5$ & V & V & V & V & V & V \\
\hline
\end{tabular}

Table 3: Topological patterns of the contour lines of the Hamiltonian (26) in the triply connected circular domains for $a=0.2, a=0.3$ and $a=0.5$ and various $b$. 


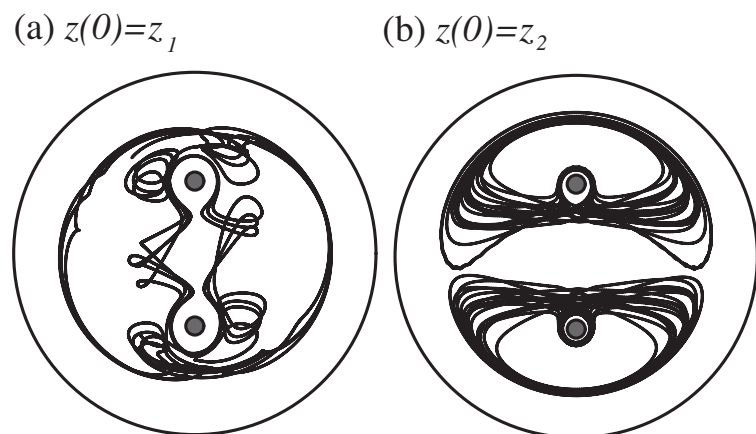

Trajectories (c) $z(0)=z_{1}$

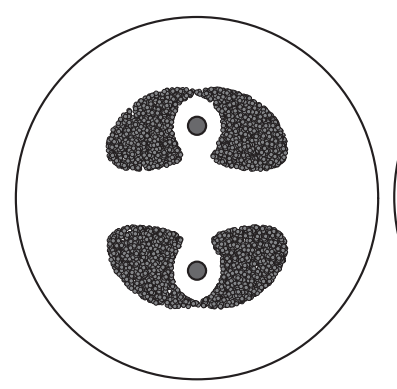

Poincare sections

(d) $z(0)=z_{2}$

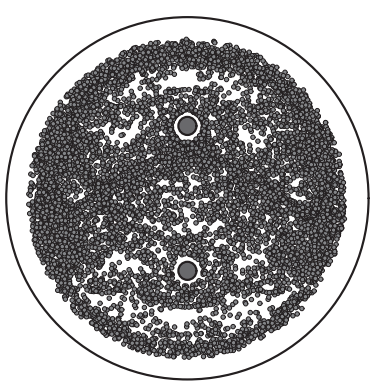

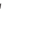

Figure 12: Motion of the two point vortices in the symmetric domain with the two obstacles (23) for $a=0, b=0.4$ and $r=0.05$. The amplitude of the perturbation in the initial configuration (28) is $\epsilon_{1}=1.0 \times 10^{-4}$. Trajectories of the two point vortices for (a) $\boldsymbol{z}(0)=\boldsymbol{z}_{1}$ and (b) $\boldsymbol{z}_{2}$, and their corresponding Poincaré sections $\left\{\operatorname{Re} z_{1}=0\right\}$ for $(\mathrm{c}) \boldsymbol{z}(0)=\boldsymbol{z}_{1}$ and $(\mathrm{d}) \boldsymbol{z}_{2}$ respectively.

equations (19) and (20) are integrated by the fourth order Runge-Kutta method with time step size $\Delta t=0.001$. We consider the same symmetric circular domain with the two obstacles (23) for $a=0, b=0.4$ and $r=0.05$ as in Figure 1(b). On the other hand, we deal with an initial configuration without the reflectional symmetry. Let $\zeta_{1}$ and $\zeta_{2}$ denote the two saddle fixed points obtained in Figure 1(b). Then the initial configuration is given by

$$
\boldsymbol{z}(0) \equiv\left(z_{1}(0), z_{2}(0)\right)=\boldsymbol{z}_{k} \equiv\left(\zeta_{k}+\epsilon_{1}(1+\mathrm{i}), \zeta_{k}^{*}+\epsilon_{2} \mathrm{i}\right) \in \mathbb{C}^{2}, \quad k=1,2,
$$

for $\epsilon_{1}$ and $\epsilon_{2} \in \mathbb{R}$. For given $\epsilon_{1}$, the parameter $\epsilon_{2}$ is determined numerically so that the value of the Hamiltonian for (28) is equivalent to that of the Hamiltonian (22) with the reflectional symmetry at $z_{1}=\zeta_{k}$ up to 12 digits.

Figure 12 shows the trajectories of the two point vortices for the initial data (a) $\boldsymbol{z}(0)=\boldsymbol{z}_{1}$ and for (b) $\boldsymbol{z}(0)=\boldsymbol{z}_{2}$ with $\epsilon_{1}=1.0 \times 10^{-4}$. Although the initial disturbance $\epsilon_{1}$ is quite small, they are far from being integrable. Figure 13(a) shows the evolutions of $d(t)=\left|z_{1}(t)-z_{2}^{*}(t)\right|$ for $\boldsymbol{z}(0)=\boldsymbol{z}_{1}$ and $z_{2}$ with $\epsilon_{1}=1.0 \times 10^{-4}$, which indicate that the reflectional symmetry between the two point vortices is breaking, since $d(t) \equiv 0$ for all time if the system is integrable. In addition, in order to see the dependence on the initial disturbance, we observe the center of the two point vortices, i.e., $c(t)=\frac{1}{2}\left(z_{1}(t)+z_{2}(t)\right)$, which evolves in the real axis if the motion is integrable. Figure 13 also shows the trajectories of $c(t)$ for $(\mathrm{b}) \boldsymbol{z}(0)=\boldsymbol{z}_{1}$ and for $(\mathrm{c}) \boldsymbol{z}(0)=\boldsymbol{z}_{2}$ with $\epsilon_{1}=1.0 \times 10^{-4}$ and $3.0 \times 10^{-4}$, which indicates that the motion of the two point vortices is sensitive to the initial disturbance, since the trajectories of $c(t)$ for $\epsilon_{1}=1.0 \times 10^{-4}$ and $3.0 \times 10^{-4}$ are different.

The motion of the two point vortices is reduced to three-dimensional dynamical system since the equations (19) and (20) define the Hamiltonian dynamical system in the four-dimensional phase space $\left(\operatorname{Re} z_{1}, \operatorname{Im} z_{1}, \operatorname{Re} z_{2}, \operatorname{Im} z_{2}\right)$ and the orbit evolves in the same energy surface of the Hamiltonian. Thus in order to observe the motion of the two point vortices from another point of view, we show the Poincaré section $\left\{\operatorname{Re} z_{1}=0\right\}$ in Figure 12(c) and (d) corresponding to the trajectories of Figure $12(\mathrm{a})$ and (b). For $\boldsymbol{z}(0)=\boldsymbol{z}_{1}$, the orbits spread in a region near the homoclinic orbits to $\zeta_{1}$. On the other hand, they spread inside the whole unit circle for $\boldsymbol{z}(0)=\boldsymbol{z}_{2}$. We infer from them that the evolution of the two point vortices without the reflectional symmetry could be chaotic. 
(a) $d(t)$

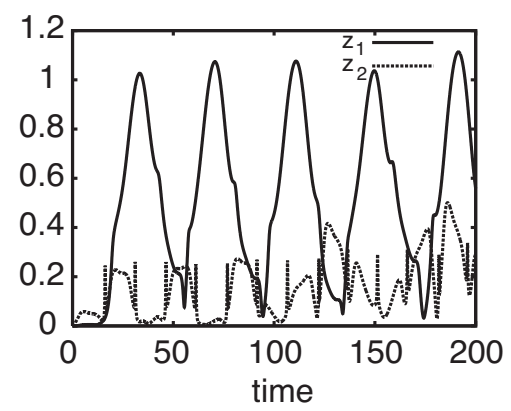

(b) $z(0)=z_{1}$

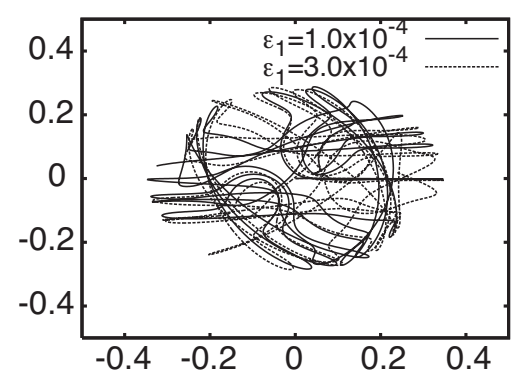

(c) $z(0)=z$

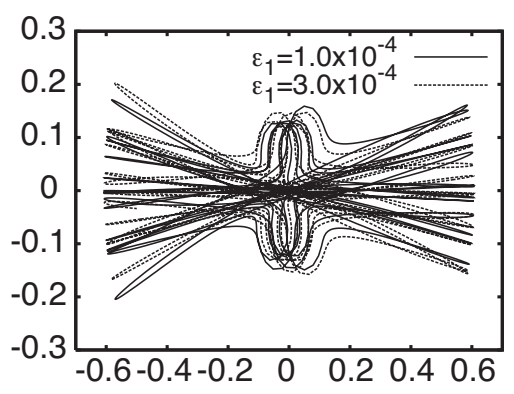

Figure 13: (a) Evolutions of $d(t)=\left|z_{1}(t)-z_{2}^{*}(t)\right|$ for the initial configurations $\boldsymbol{z}(0)=\boldsymbol{z}_{1}$ and $\boldsymbol{z}_{2}$ with $\epsilon_{1}=1.0 \times 10^{-4}$. Trajectories of the center $c(t)$ of the two point vortices for (b) $\boldsymbol{z}(0)=\boldsymbol{z}_{1}$ and for $(\mathrm{c}) \boldsymbol{z}(0)=\boldsymbol{z}_{2}$ with amplitudes $\epsilon_{1}=1.0 \times 10^{-4}$ and $3.0 \times 10^{-4}$ in (28).

\section{Summary and concluding remarks}

We have derived the equation of motion for the $N$ point vortices in a multiply connected domain, called the circular domain, inside the unit circle that has $M$ circular obstacles. The interaction between the point vortices is expressed by the function $\omega_{\zeta}(\zeta, \alpha) / \omega(\zeta, \alpha)$, in which $\omega(\zeta, \alpha)$ is the Schottky-Klein prime function associated with the circular domain. Thanks to the explicit representation, it is possible to approximate the solution of the Euler equations in the circular domain numerically with the vortex method[2]. Moreover, for given $N$ point vortices $\left\{z_{\lambda} \mid \lambda=1, \ldots, N\right\}$ with the strengths $\left\{\Gamma_{\lambda} \mid \lambda=1, \cdots, N\right\}$, we have the following expression for the instantaneous velocity field at any point $z$ in the circular domain,

$$
\boldsymbol{u}(z)=\frac{\mathrm{i}}{2 \pi} \sum_{\lambda=1}^{N} \Gamma_{\lambda}\left(\frac{\omega_{\zeta}^{*}\left(z^{*}, z_{\lambda}^{*}\right)}{\omega^{*}\left(z^{*}, z_{\lambda}^{*}\right)}-\frac{\omega_{\zeta}^{*}\left(z^{*}, z_{\lambda}^{-1}\right)}{\omega^{*}\left(z^{*}, z_{\lambda}^{-1}\right)}\right)
$$

which is available to compute the motion of passive scalars advected by the $N$ point vortices.

Let us mention the equation for the $N$ point vortices for general multiply connected domains. Suppose that the conformal mapping $\zeta=f(w)$ from a given multiply connected domain $\mathcal{D}_{w}$ in the complex $w$-plane to a circular domain $\mathcal{D}_{\zeta}$ in the complex $\zeta$-plane is constructed. Then the motion of the $N$ vortex points $\left\{w_{\lambda} \mid \lambda=1, \ldots, N\right\}$ in $\mathcal{D}_{w}$ is described by a Hamiltonian dynamical system, whose Hamiltonian $H^{(w)}$ is represented by

$$
H^{(w)}\left(w_{1}, w_{1}^{*}, \ldots, w_{N}, w_{N}^{*}\right)=H^{(\zeta)}\left(\zeta_{1}, \zeta_{1}^{*}, \ldots, \zeta_{N}, \zeta_{N}^{*}\right)-\sum_{\lambda=1}^{N} \frac{\Gamma_{\lambda}^{2}}{4 \pi} \log \left|f_{w}\left(w_{\lambda}\right)\right|,
$$

in which $H^{(\zeta)}$ denotes the Kirchhoff-Routh function for the $N$ point vortices $\left\{\zeta_{\lambda} \mid \lambda=1, \ldots, N\right\}$ in the circular domain $\mathcal{D}_{\zeta}$ with $\zeta_{\lambda}=f\left(w_{\lambda}\right)$.

We have applied the equation to investigate the motion of two point vortices with the unit strength of the opposite signs. When the domain is either the simply connected circle or the double connected concentric annuls, their motion is integrable. On the other hand, for the circular domains with more than one obstacle, it is not integrable in general. However, when the circular domain is symmetric with respect to the real axis, the motion of the two point vortices is reduced to that of a single point vortex in the upper semi-circle, if their initial configuration has 
the same symmetry. We have described the motion of the single point vortex when the circular domain contains two, three and four obstacles by plotting the contour lines of the Hamiltonian. We focus on the topological pattern in the semi-circle that consists of fixed configurations, homoclinic/heteroclinic orbits and the obstacles. Then we describe the transitions and bifurcations between the topological patterns, which occur due to the pitchfork bifurcation, the degenerate pinching bifurcations of the contour line around the center point and the reconnection of the heteroclinic/homoclinic orbits. Comparing the results with the motion of the single point vortex in the circular domains with the same connectivity, we find that more topological patterns of the contour lines and more complicated transitions and bifurcations between them are possible for the semi-circles than for the circles. Moreover, we give several numerical examples of the motion of the two point vortices when the saddle points in the integrable system are slightly perturbed. Although the initial perturbation is quite small, the evolution of the two point vortices without the reflectional symmetry becomes complicated, which is sensitive to the initial amplitude of disturbance. Poincaré section for their trajectories illustrates that the motion of the two point vortices becomes chaotic.

\section{Acknowledgements}

This study is partially supported by JSPS grant \#19654014 and by JST PRESTO. I would like to show my gratitude to School of Applied Mathematics at University of Sheffield for providing me with nice research environments during the stay as a visiting scholar.

\section{References}

[1] H. Baker, Abelian functions, 1995, Cambridge Univ. Press, Cambridge.

[2] G. H. Cottet and P.D. Koumoutsakos, Vortex Methods: Theory and Practice, 2000, Cambridge Univ. Press, Cambridge.

[3] D. Crowdy and J. Marshall, Analytical formulae for the Kirchhoff-Routh path function in multiply connected domains, Proc. Roy. Soc. A, 461 (2005), pp.2477-2501.

[4] D. Crowdy and J. Marshall, The motion of a point vortex around multiple circular islands, Phys. Fluids, 17 (2005) 056602.

[5] D. Crowdy, Analytical solutions for uniform potential flow past multiple cylinders, Euro. J. Mechanics B/Fluids, 25 (2006), pp.459-470.

[6] D. Crowdy, Calculating the lift on a finite stack of cylindrical aerofoils, Proc. Roy. Soc. A, 462 (2006), pp.1387-1407.

[7] M. Flucher and B. Gustafsson, Vortex motion in two dimensional hydrodynamics, TRITAMAT-1997-MA 02. Stockholm: Royal Institute of Technology.

[8] C. C. Lin, On the motion of vortices in two dimensions. I. Existence of the Kirchhoff-Routh function, Proc. Natl Acad. Sci., 27 (1941), pp. 570-575.

[9] C. C. Lin, On the motion of vortices in two dimensions. II. Some further investigations on the Kirchhoff-Routh function. Proc. Natl Acad. Sci., 27 (1941), pp. 575-577. 
[10] Z. Nehari, Conformal mapping, 1952, McGraw-Hill, New York.

[11] P. K. Newton, The N-vortex problem, Analytical techniques, Springer-Verlag, New York, 2001.

[12] P. G. Saffman, Vortex Dynamics, 1992, Cambridge Univ. Press, Cambridge. 